\title{
Effects of a dipolar field in the spin dynamics of a Fermi liquid
}

\author{
P. L. Krotkov用 \\ L. D. Landau Institute for Theoretical Physics, Russian Academy of Sciences, 2 Kosygina st., 117334 Moscow, Russia \\ V. P. Mineev \\ Départament de Recherche Fondamentale sur la Matière Condensée, \\ Commisariat à l'Énergie Atomique, 17 rue des Martyrs, 38054 Grenoble Cedex 9, France
}

G. A. Vermeulen

Centre des Recherches sur les Très Basses Températures, laboratoire associé à l'Université Joseph Fourier, CNRS, BP 166, Grenoble Cédex 9, France

(Dated: January, 20, 2001)

\begin{abstract}
We study spin dynamics of a normal Fermi liquid taking into account the demagnetizing field produced by the spin system itself. Linear solutions of the spin dynamics equations in the form of standing spin waves in a finite volume of liquid are found. At almost all known experimental conditions the influence of demagnetizing field can be satisfactorily described by the first order of perturbation theory. We carried out perturbational calculations for two geometries of experimental cell - spherical and finite-cylindrical. We performed also exact numerical simulations of the spin wave spectra in a spherical cell at an arbitrary strength of the demagnetizing field.

The obtained results are applied in particular to conditions of recent experiment (G.Vermeulen and A.Roni, Phys. Rev. Lett. 86, 248 (2001)) related to the problem of zero temperature transverse relaxation in a polarized Fermi liquid. We found that not taking into account demagnetizing field leads to negligible errors in the measured relaxation time, thus supporting the conclusion of the absence of zero temperature spin wave damping.
\end{abstract}

PACS numbers: 67.57.Lm, 67.60.-g, 67.65.+z, 67.80.Jd, 75.30.Ds

\section{INTRODUCTION}

Spin dynamics of a strongly spin-polarized normal Fermi liquid still captures appreciable theoretical and experimental interest. Among the main questions here is whether the transverse (i.e. in a direction perpendicular to the external field) magnetization excitations are damped at zero temperature.

Polarizing a Fermi liquid creates a gap $\sim \hbar \gamma M / \chi_{n}$ between the two Fermi energies for spins up and down. Here $\gamma$ is the gyromagnetic ratio of ${ }^{3} \mathrm{He}$ nuclei, $\chi_{n}$ is the susceptibility of the liquid. Meyerovich has pointed out [1] that the existence of the gap leads to a nonconventional temperature dependence of the transverse relaxation time, $\propto\left(T^{2}+T_{\mathrm{a}}^{2}\right)^{-1}$, where $T_{\mathrm{a}}$ is of the order of the gap, and therefore to a damping of the transverse excitations even at zero temperature. This idea has been pursued in several theoretical papers [2], [3], 细 and was contested recently by Fomin [5], who argued that the conclusion of existence of zero-temperature attenuation is drawn from the wrong premises about the ground state of a polarized Fermi-liquid, viz., from treating the quasiparticles between two Fermi levels as excitations. Whereas as long as the polarization of the liquid is conserved, these particles should be considered as an inalienable part of

\footnotetext{
*Electronic address: krotkov@itp.ac.ru

${ }^{\dagger}$ Electronic address: mineev@drfmc.ceng.cea.fr

\#lectronic address: overmeul@labs_polycnrs-gre.fr; URL: http: //www-crtbt.polycnrs-gre.fr/he3pol
}

the ground state.

Curiously, a similar discussion arose in mesoscopic physics where a poorly argued concept of finite dephasing time at $T=0$ has been developed [11] and contested [12], 13].

The results of the measurements of the spin diffusion coefficients by spin echo experiments in pure ${ }^{3} \mathrm{He}$ [6], [7] and in solutions of ${ }^{3} \mathrm{He}$ in ${ }^{4} \mathrm{He}[8$, , 9] with a spin polarization of a few percent revealed a finite value of $T_{\mathrm{a}}$ in a qualitative agreement with the zero-attenuation concept. But the observed $T_{\mathrm{a}}$ were several times greater than theoretical estimations in [2], [3], 泪. On the contrary, the recent measurements of linear spin wave damping in dilute ${ }^{3} \mathrm{He}$ at even higher polarizations [10] are in agreement with Fomin's theory $\left(T_{\mathrm{a}}=0\right)$, although the upper limit for a finite $T_{\mathrm{a}}$ set by this experiment (due to the error bars) does not allow to rule out completely the existing theory of zero temperature spin wave damping.

A coherent theory of strongly polarized Fermi liquids based on a properly defined ground state is lacking. On the other hand a proper interpretation of the experimental data for a strongly polarized liquid is itself non-trivial.

The point is that the magnetic field acting on the spins of a liquid is conventionally supposed to equal the external field $\mathbf{H}^{e}$. In reality the field inside a specimen is well-known to differ from $\mathbf{H}^{e}$ due to the shape-dependent demagnetizing field proportional to the magnetization. An oscillating magnetization thus acts back on itself via the demagnetizing (or dipolar) field. This phenomenon manifests itself as magnetostatic waves in ferrimagnets theoretically described by Kittel [14] and Walker [15]. 
Walker originally showed that if the magnetization $\mathbf{M}$ is supposed to obey the Larmor precession around the internal magnetic field $\mathbf{H}^{i}$

$$
\left(\partial_{t}+\gamma \mathbf{H}^{i} \times\right) \mathbf{M}=0,
$$

then because $\mathbf{M}$ and $\mathbf{H}^{i}$ are related through Maxwell equations they can self-consistently oscillate only with certain frequencies localized in the range $\sim 2 \pi \gamma M$ near the Larmor frequency.

In an interacting Fermi liquid (11) should be replaced with the Leggett system of equations. In the linear approximation, solutions of this system are standing spin wave modes with the widths proportional to the transverse relaxation time. So study of the behavior of the widths of the modes with temperature is one of the possible ways to detect the zero-temperature transverse attenuation.

For weakly-polarized liquids the effects of the demagnetizing field can be discarded. But it is preferable to have strong polarizations in order to increase the predicted temperatures of the attenuation onset. So the effect of polarization has to be taken into consideration for the proper interpretation of the spectra. On the other hand at sufficiently strong polarizations one can expect considerable changes in the Leggett description of the Fermi liquid spin dynamics. In particular, due to the presence of two Fermi surfaces a double set of the Fermi-liquid parameters must enter the theory. We will use Leggett equations assuming that they are still valid when the share of polarized nuclei of the liquid does not surpass $\sim 10 \%$.

To include dipolar field we have chosen to write out the dipolar part of the internal field $\mathbf{H}^{i}$ explicitly as an integral of the magnetization, this integral being a general solution of Maxwell differential equations with Maxwell boundary conditions. Thus we work with a closed integro-differential equation directly on the magnetization.

A short review of other possible methods is done in the Discussions section.

We start directly from the generalized Leggett equations and study the effects of the demagnetizing field coherently. We specialize to the case of linear spin waves, setting spin echo experiments aside.

A full-blown study including numerical calculations is done only for a spherical shape. For a finite cylinder as well as for a sphere we also calculated the changes to the spectra by the demagnetizing field using perturbation theory.

Our results show that the demagnetizing field introduces small corrections (about 4\%) to the value of low temperature transversal relaxation rate experimentally determined by G.Vermeulen and A.Roni [10]. Hence, the main conclusion of Ref. [10] about the absence of zerotemperature spin wave damping is supported.

The paper is organized as follows. Sections II and III form the basis needed to comprehend the authors' point of view. In Sec. II we show how to include the dipolar field in the standard Leggett equations and to how to linearize the result to obtain an equation for spin waves subject to both exchange and demagnetizing fields.

In Sec. III as the simplest application of the theory developed we find corrections to the spin wave spectra in a finite cylinder in the first order of perturbation theory. At the end of the Sec. III we find dipolar limitations on the correct determination of the transverse relaxation time from the conventional interpretation of the spectra.

The next Section IV contains similar first order perturbational estimation of the dipolar-field corrections to the spin waves spectra in a spherical container.

The results for a sphere are compared to numerical simulations carried out in Section V, where we also calculate spin wave spectra in the regimes of intermediate and strong demagnetizing fields.

In the last Section VI we discuss conclusions.

\section{STATEMENT OF THE PROBLEM}

\section{A. Basic equations}

Spin dynamics of a Fermi liquid is described by the Leggett coupled system of two partial differential equations on the local magnetization $\mathbf{M}(\mathbf{r}, t)$ and its current $\mathbf{J}_{i}(\mathbf{r}, t)$ [20]

$$
\begin{aligned}
\left(\partial_{t}+\gamma \mathbf{B} \times\right) & \mathbf{M}+\partial_{i} \mathbf{J}_{i}=0 \\
\left(\partial_{t}+\gamma \mathbf{B} \times\right) & \mathbf{J}_{i}+\frac{w^{2}}{3} \partial_{i}\left(\mathbf{M}-\mathbf{M}_{0}\right) \\
& +\kappa \frac{\gamma}{\chi_{n}} \mathbf{M} \times \mathbf{J}_{i}=-\frac{\mathbf{J}_{i}}{\tau_{1}} .
\end{aligned}
$$

Here $\mathbf{B}$ is the flux density inside the sample [21], $w^{2}$ the renormalized Fermi velocity $w^{2}=v_{F}^{2}\left(1+F_{0}^{a}\right)\left(1+F_{1}^{a} / 3\right)$ and $\tau_{1}$ the renormalized relaxation time $\tau_{1}=\tau /(1+$ $\left.F_{1}^{a} / 3\right) . \quad F_{0}^{a}$ and $F_{1}^{a}$ are the coefficients of expansion of the antisymmetric part of the Fermi-liquid interaction in the spherical harmonics. The equilibrium magnetization is

$$
\mathbf{M}_{0}=\chi_{n} \mathbf{H}^{i},
$$

where $\mathbf{H}^{i}=\mathbf{B}-4 \pi \mathbf{M}$ is the internal field.

Spin dynamics equations of a Fermi liquid reduce to the form (2), (3) in either of the regimes - collisionless $C \gg$ 1 or hydrodynamic $C \ll 1$, where the regime parameter

$$
C=\kappa\left(\gamma H^{i}\right)\left(M / M_{0}\right) \tau_{1}
$$

The factor $M / M_{0}$ accounts for the possibility for the polarization $M$ be higher than the equilibrium value $M_{0}-$ in experiments [10], |18 $M / M_{0}$ varied from 1 to 5 .

The condition of applicability of the Leggett equations [20] is that the characteristic scale of spatial inhomogeneity $\xi$ be greater than the quasiparticle mean free path $v_{F} \tau$ or the magnetic length $v_{F} \tau / C$ which one is the shorter,

$$
\xi \gg \min \left\{v_{F} \tau, v_{F} \tau / C\right\} .
$$


In the collisionless regime $(C \gg 1)$ the magnetic length $v_{F} \tau / C$ is the shorter and thus should be smaller than $\xi$. While in the hydrodynamic regime $(C \ll 1)$ the spatial scale $\xi$ should exceed the mean free path $v_{F} \tau$.

Eq. (3) contains the torque due to the local molecular field $\kappa \mathbf{M}(\mathbf{r}, t) / \chi_{n}$ acting on current $\mathbf{J}_{i}(\mathbf{r}, t)$. The combination of the Fermi-liquid constants $F_{0}^{a}$ and $F_{1}^{a}$

$$
\kappa=\frac{\frac{1}{3} F_{1}^{a}-F_{0}^{a}}{1+F_{0}^{a}}
$$

measures the strength of the exchange interaction. It vanishes when turning the exchange off.

Leggett originally 20 considered the case of a weaklypolarized sample, wherein $\mathbf{B}=\mathbf{H}^{e}$ - the external magnetic field at infinity. Generally, the relation between $\mathbf{B}$ and $\mathbf{H}^{e}$ is to be determined from the conventional boundary value problem of solving the magnetostatic equations in a non-conducting medium

$$
\partial \times \mathbf{H}=0, \quad \partial \mathbf{B}=0
$$

with Maxwell boundary conditions of the continuity of $B_{n}$ and $\mathbf{H}_{t}$ at the boundary of the sample and of $\mathbf{H} \rightarrow \mathbf{H}^{e}$ at infinity. In (8)

$$
\mathbf{H}=\mathbf{B}-4 \pi \mathbf{M}
$$

A general formal solution of (8), (9) satisfying the appropriate boundary conditions is 22

$$
\mathbf{H}=\mathbf{H}^{e}+\mathbf{H}_{\mathrm{dip}}
$$

where

$$
\mathbf{H}_{\mathrm{dip}}(\mathbf{r})=\boldsymbol{\partial}\left(\boldsymbol{\partial} \int \frac{\mathbf{M}\left(\mathbf{r}^{\prime}\right)}{\left|\mathbf{r}-\mathbf{r}^{\prime}\right|} d^{3} \mathbf{r}^{\prime}\right)
$$

is called the dipolar field. It is straightforward to verify that Eqs. (10) and (9) are indeed the solution in the whole space with the help of

$$
\partial^{2}\left|\mathbf{r}-\mathbf{r}^{\prime}\right|^{-1}=-4 \pi \delta\left(\mathbf{r}-\mathbf{r}^{\prime}\right)
$$

$\mathbf{M}$ in its turn has to be found from the Leggett equations (2), (3). Therefore, the closed system of integrodifferential Eqs. (2), (3), (9)-(11) completely describes normal Fermi liquid electrodynamics with the effects of both inhomogeneity and the demagnetizing field taken into account.

Inside the sample the field $\mathbf{H}$ from $\sqrt{10}$ is called $\mathbf{H}^{i}$ - the internal magnetic field. The difference between external field at infinity $\mathbf{H}^{e}$ and $\mathbf{H}^{i}$ is usually denoted as

$$
\mathbf{H}^{i}-\mathbf{H}^{e}=-4 \pi \underline{\widehat{n}}[\mathbf{M}]
$$

where the (tensor) operator $\underline{\underline{x}}$, acting by the rule

$$
\underline{\widehat{n}}[\mathbf{M}]=-\frac{1}{4 \pi} \partial\left(\partial \int_{V} \frac{\mathbf{M}\left(\mathbf{r}^{\prime}\right)}{\left|\mathbf{r}-\mathbf{r}^{\prime}\right|} d^{3} \mathbf{r}^{\prime}\right)
$$

is called demagnetizing operator. Let us agree to denote an operator with a hat over a letter and a tensor with an underlined letter. The demagnetizing tensor operator $\underline{\hat{n}}$ is specific to the shape of the sample, over volume of which the integration in (14) is taken, and generally is coordinate-dependent. It only reduces to the tensor of constant demagnetizing coefficients $\underline{n}$ when acting on spatially homogeneous distributions in ellipsoidal samples (including limiting cases of a slab and an infinite cylinder).

\section{B. Static magnetization distribution}

In a linear spin wave the magnetization rotates with a small amplitude $\mathbf{m}$ around its (large) stable value $\mathbf{M}$ in a static external magnetic field $\mathbf{H}^{e}=H^{e} \hat{z}$.

Consider a general static $\left(\partial_{t} \mathbf{M}=0, \mathbf{J}_{i}=0\right)$ case. In order for $\mathbf{J}_{i}=0$ there should be

$$
\partial_{i}\left(\mathbf{M}-\mathbf{M}_{0}\right)=0
$$

and then for $\partial_{t} \mathbf{M}=0$ the magnetization should be locally directed along $\mathbf{H}^{i}$

$$
\mathbf{M}(\mathbf{r})=\chi_{n} A(\mathbf{r}) \mathbf{H}^{i}(\mathbf{r})
$$

The function $A(\mathbf{r})$ must be such that $\mathbf{M}(\mathbf{r})$ satisfies (15). This imposes restrictions on the spatial dependence of $A(\mathbf{r})$, but leaves $A(0)$ arbitrary.

So there exists continuum of static non-equilibrium magnetization distributions numbered by $A \equiv A(0)$. It is this $A$ which is represented as $M / M_{0}$ in Table $\mathrm{I}$.

In equilibrium (4) $A(\mathbf{r}) \equiv 1$.

To find a form of a non-equilibrium static magnetization distribution, we shall use the smallness of $\chi_{n}$. For pure ${ }^{3} \mathrm{He}$, the magnetic susceptibility is

$$
\chi_{n}=\hbar^{2} \gamma^{2} N_{0} / 2\left(1+F_{0}^{a}\right) \sim 10^{-7},
$$

where $N_{0}=m^{*} k_{F} / 2 \pi^{2} \hbar^{2}$ is the density of states on the Fermi surface. For ${ }^{3} \mathrm{He}-{ }^{4} \mathrm{He}$ mixtures $\chi_{n}$ is less, proportional to $k_{F} \propto \sqrt[3]{x}$, where $x$ is the concentration of ${ }^{3} \mathrm{He}$ atoms in the mixture.

Substituting (16) into (13) yields

$$
\mathbf{H}^{i}(\mathbf{r})=\mathbf{H}^{e}(\mathbf{r})-4 \pi \chi_{n} \underline{\widehat{n}}\left[A(\mathbf{r}) \mathbf{H}^{e}(\mathbf{r})\right]+O\left(\chi_{n}^{2}\right) .
$$

For the reasons that will become clear below the external magnetic field is taken almost constant, with a small gradient along its direction

$$
\mathbf{H}^{e}(\mathbf{r})=\mathbf{H}^{e}\left(1+z \nabla \omega_{L} / \omega_{L}\right)
$$

The presence of the field gradients in the perpendicular directions, necessary for the fulfillment of the condition $\partial \mathbf{H}^{e}=0$, is inessential for the following discourse.

From (15) it then follows that $\nabla A=(1-A) \nabla \omega_{L} / \omega_{L}$, i.e. the spatial inhomogeneity of $A(\mathbf{r})=A+z \nabla A$ has the same smallness. 
Leaving in (18) only the first order terms in either $\chi_{n}$ or $z \nabla \omega_{L} / \omega_{L}$, we have

$$
\gamma \mathbf{H}^{i}=\left(\omega_{L}+z \nabla \omega_{L}\right) \hat{z}-\omega_{M} \underline{\hat{\widehat{x}}}[\hat{z}] .
$$

Here

$$
\omega_{L}=\gamma H^{e}
$$

is the Larmor frequency and

$$
\omega_{M}=4 \pi \gamma M=4 \pi \chi_{n} A \omega_{L}
$$

is the frequency corresponding to magnetization. $\underline{\widehat{n}}[\hat{z}]$ is generally a coordinate dependent vector. For ellipsoidal samples it reduces to $\underline{n} \hat{z}$. If one of the principal axes of the ellipsoid (which are also the principal axes of the demagnetizing coefficients tensor $\underline{n}$ ) coincides with $\hat{z}$, we have $\underline{n} \hat{z}=\hat{z} n^{(z)}$, where $n^{(z)}$ is the $z$-th demagnetizing coefficient. E.g., for a sphere $n^{(z)}=\frac{1}{3}$, for a plane-parallel slab with the edges perpendicular to $\widehat{z}$ the coefficient $n^{(z)}=1$, and for an infinite circular cylinder with the generatrix parallel to $\widehat{z}$ the coefficient $n^{(z)}=0$.

The small ratio $2 R \nabla \omega_{L} / \omega_{L}$, where $2 R$ is the sample size, together with $4 \pi \chi_{n} A(0) \sim 10^{-6}$ are the two small parameters in the problem. Conventionally the dipolar field is not taken into account and the second parameter is considered negligibly small. This is no longer justified for recent experimental conditions as is seen from Table $\mathrm{I}$, where the ratio of the second parameter to the first

$$
\omega_{M} / 2 R \nabla \omega_{L}
$$

is represented for various experimental conditions.

\section{Linearized equations of motion}

To obtain the linearized form of the equations of motion (2), (3) we expand all the macroscopic quantities near their stationary values:

$$
\begin{aligned}
\mathbf{H}^{e}=H^{e} \widehat{z}+\mathbf{h}^{e}, & \mathbf{H}^{i}=\mathbf{H}^{i}+\mathbf{h}^{i}, \\
\mathbf{M}=\mathbf{M}+\mathbf{m}, & \mathbf{J}_{i}=\mathbf{j}_{i},
\end{aligned}
$$

where the static value of $\mathbf{H}^{i}$ is that from (20). The radio frequency field $\mathbf{h}^{e}$ plays the role of a driving force for the spin system response $\mathbf{m}$. We denote $\mathbf{h}^{i}=\mathbf{h}^{e}-4 \pi \underline{\widehat{n}}[\mathbf{m}]$.

The static $\mathbf{M}$ in (24) is the result of the substitution of $(20)$ into $(16)$

$$
\gamma \mathbf{M} / \chi_{n}=\omega_{L}\left(A+z \nabla \omega_{L}\right) \hat{z}-\omega_{M} A H^{e} \underline{\hat{n}}[\hat{z}] .
$$

However, we can retain only the greatest term in $\mathbf{M}$

$$
\mathbf{M}=\chi_{n} A \mathbf{H}^{e}
$$

when linearizing Eqs. (2), (3). In (2) this is simply due to the fact that the terms of $\mathbf{M}$ to be omitted are of the order $O\left(\chi_{n} z \nabla \omega_{L} / \omega_{L}\right)$ and $O\left(\chi_{n}^{2}\right)$. In (3) $\mathbf{M}$ is divided by $\chi_{n}$ and the justification is lengthier. We will suppose
(26) and discuss why only the main term in $\mathbf{M}$ should be left after the derivation below.

In practice one usually is interested in movements quasistationary in the Larmor frame. To a first approximation one supposes that $\mathbf{j}_{i}(\mathbf{r}, t)$ is stationary, i.e. precesses with the frequency $\gamma \mathbf{B}:\left(\partial_{t}+\gamma \mathbf{B} \times\right) \mathbf{j}_{i}=0$. Then resolving Eq. (3) with respect to $\mathbf{j}_{i}$ with $\mathbf{M}$ from (26) gives

$$
\mathbf{j}_{i}=-\frac{D_{0}}{1+C^{2}}\left[\partial_{i} \mathbf{m}-C \widehat{z} \times \partial_{i} \mathbf{m}+C^{2} \widehat{z}\left(\widehat{z} \partial_{i} \mathbf{m}\right)\right],
$$

where the diffusion coefficient $D_{0}=\frac{1}{3} w^{2} \tau_{1}=\frac{1}{3} v_{F}^{2} \tau(1+$ $\left.F_{0}^{a}\right)$ and

$$
C=\kappa \tau_{1} \gamma M / \chi_{n}=\kappa \tau_{1} A \omega_{L}
$$

is another expression for the regime parameter (5).

One then plugs the divergence of (27) into (2). The divergence of $\mathbf{j}_{i}$ has the order

$$
\partial_{i} \mathbf{j}_{i} \sim D_{0} \partial^{2} \mathbf{m} \sim\left(D_{0} / \xi^{2}\right) \mathbf{m},
$$

where $\xi \sim \sqrt[3]{D_{0} / \nabla \omega_{L}}$ is the characteristic scale (49). So $\partial_{i} \mathbf{j}_{i} \sim\left(\xi \nabla \omega_{L}\right) \mathbf{m}$ has already the smallness $z \nabla \omega_{L} / \omega_{L}$. If we had accounted for the higher order terms in $\mathbf{M}$ than (26) when calculating the current, these terms would have entered $\mathbf{j}_{i}$ through the regime parameter $C$, and after multiplication with $D_{0} \partial^{2} \mathbf{m}$ would have produced terms of the order $O\left(\chi_{n} z \nabla \omega_{L} / \omega_{L}\right) \mathbf{m}$ and $O\left(\chi_{n}^{2}\right) \mathbf{m}$. That is why we were allowed to substitute simply (26) in (3).

In the linear approximation $\mathbf{m}(\mathbf{r})$ is in each point perpendicular to the static $\mathbf{M}(\mathbf{r})$ if the absolute value of the magnetization is conserved. Exp. (25) shows that apart from the major $\hat{x}$ - and $\hat{y}$ - components $\mathbf{m}$ also has a minor $m_{z} \sim \chi_{n} m_{x}$. This component also may be seen to give higher order terms and is therefore negligible.

We will thus consider $\mathbf{m} \perp \widehat{z}$. The last term on the right-hand side of (27) then vanishes, and substituting (27) into (2) one gets

$$
\left(\partial_{t}-\frac{D}{C} \partial^{2}\right) \mathbf{m}+\widehat{z} \times\left(\gamma H_{z}^{i} \mathbf{m}+D \partial^{2} \mathbf{m}-\gamma M \mathbf{h}^{i}\right)=0 .
$$

Here we left only the $\hat{z}$-component of $\mathbf{H}^{i}$ because $\mathbf{H}_{\perp}^{i}$, which multiplies vectorially only by $m_{z} \hat{z}$, produces terms $O\left(\chi_{n}^{2}\right)$.

In (30) we introduced the effective spin diffusion coefficient

$$
D=D_{0} C /\left(1+C^{2}\right) .
$$

In the strong $(C \gg 1)$ collisionless regime $D \approx$ $w^{2} / 3 \kappa \omega_{L} A$ is temperature independent.

Suppose $\mathbf{m}, \mathbf{h}$ depend on time as monochromatic waves $\mathbf{m}, \mathbf{h} \propto e^{-i \omega t}$. Then written out in components the equation of motion for $\mathbf{m}$ becomes

$$
-i \widehat{\omega}\left(\begin{array}{c}
m_{x} \\
m_{y}
\end{array}\right)+\widehat{\omega}_{L}\left(\begin{array}{c}
-m_{y} \\
m_{x}
\end{array}\right)=\frac{\omega_{M}}{4 \pi}\left(\begin{array}{c}
-h_{y}^{i} \\
h_{x}^{i}
\end{array}\right),
$$

where we designated by $\widehat{\omega}_{L}$ and $\widehat{\omega}$ respectively the operators

$$
\widehat{\omega}_{L}=\gamma H_{z}^{i}+D \partial^{2}, \quad \widehat{\omega}=\omega-i \frac{D}{C} \partial^{2} .
$$


Multiplying by

$$
\left(\begin{array}{cc}
0 & 1 \\
-1 & 0
\end{array}\right)
$$

results in the second order inhomogeneous partial differential equation $\underline{\chi}_{\omega}^{-1} \mathbf{m}=\mathbf{h}_{\perp}^{i}$, or

$$
\underline{\widehat{\chi}}_{\omega}^{-1} \mathbf{m}+4 \pi \underline{\widehat{n}}[\mathbf{m}]=\mathbf{h}_{\perp}^{e} .
$$

Here $\underline{\chi}_{\omega}^{-1}$ is the inverse susceptibility tensor

$$
\underline{\widehat{\chi}}_{\omega}^{-1}=\frac{4 \pi}{\omega_{M}}\left(\begin{array}{cc}
\widehat{\omega}_{L} & -i \widehat{\omega} \\
i \widehat{\omega} & \widehat{\omega}_{L}
\end{array}\right)
$$

To close the boundary value problem one must impose some appropriate boundary conditions on $\mathbf{m}$. Supposed that the container is made from a non-magnetic material, there is no magnetization current into the walls, and we get boundary condition in the form

$$
\left.\hat{n}_{i} \partial_{i} \mathbf{m}\right|_{\partial}=0
$$

Here $\hat{n}_{i}$ is a unit normal to the wall.

In the normal variables $m_{ \pm}=m_{x} \pm i m_{y}$ Eq. (34) has equivalent form

$$
\left(\widehat{\omega}_{L} \mp \widehat{\omega}\right) m_{ \pm}=\frac{\omega_{M}}{4 \pi}\left(h_{ \pm}^{e}+\partial_{ \pm} \boldsymbol{\partial} \int \frac{\mathbf{m}\left(\mathbf{r}^{\prime}\right)}{\left|\mathbf{r}-\mathbf{r}^{\prime}\right|} d^{3} \mathbf{r}^{\prime}\right)
$$

whence we estimate

$$
m_{-} \sim\left(\omega-\omega_{L}\right) m_{+} / 2 \omega_{L} \ll m_{+} .
$$

We see that in the vicinity of the Larmor frequency, when $\omega \simeq \omega_{L}$, the counter-rotating component $m_{-}$may be neglected with respect to the co-rotating $m_{+}$. Then (34) simplifies to a single linear inhomogeneous integrodifferential equation

$$
(\widehat{\mathcal{H}}-\omega) m_{+}(\mathbf{r})=\frac{\omega_{M}}{4 \pi} h_{+}^{e}(\mathbf{r})
$$

with a generally non-Hermitian Hamiltonian operator

$$
\begin{aligned}
\widehat{\mathcal{H}} m_{+} & =D\left(1+\frac{i}{C}\right) \partial^{2} m_{+}+\gamma H_{z}^{i}(\mathbf{r}) m_{+} \\
& -\frac{\omega_{M}}{8 \pi} \partial_{+} \partial_{-} \int \frac{m_{+}\left(\mathbf{r}^{\prime}\right)}{\left|\mathbf{r}-\mathbf{r}^{\prime}\right|} d^{3} \mathbf{r}^{\prime} .
\end{aligned}
$$

We may use the equivalence $\partial_{+} \partial_{-}=\partial^{2}-\partial_{z}^{2},(20)$ and the property (12) to rewrite the Hamiltonian as

$$
\begin{aligned}
\widehat{\mathcal{H}} m_{+} & =D\left(1+\frac{i}{C}\right) \partial^{2} m_{+}+\omega_{L}(\mathbf{r}) m_{+} \\
& +\frac{\omega_{M}}{2}\left(\left(1-2 \underline{\widehat{x}}_{z z}[1]\right) m_{+}-\underline{\widehat{n}}_{z z}\left[m_{+}\right]\right) .
\end{aligned}
$$

Here the integro-differential operator

$$
\underline{\underline{x}}_{z z}[f]=-\frac{1}{4 \pi} \partial_{z}^{2} \int_{V} \frac{f\left(\mathbf{r}^{\prime}\right)}{\left|\mathbf{r}-\mathbf{r}^{\prime}\right|} d^{3} \mathbf{r}^{\prime}
$$

is the $z z$-component of the demagnetizing tensor (14). And $\underline{\widehat{a}}_{z z}[1]$ is a scalar function on coordinates, which actually coincides with the $z$-th demagnetizing coefficient $n^{(z)}$ for ellipsoids with one of the principal axes parallel to $\hat{z}$. $\omega_{L}(\mathbf{r})$ denotes $\omega_{L}+z \nabla \omega_{L}$.

To conclude, we have derived equations of motion (34) or (38) for small deviations of magnetization from static values. The magnetization as a function of external rf field is a response of the system on a particular radio frequency $\omega$. The full form of the equations of motion (34) is unnecessarily complicated because it contains superfluous information on the dynamics of the counter-rotating component $m^{-}$of the magnetization. An example of solution of the full Eq. (34) for an infinite medium in a uniform magnetic field is analyzed in Appendix A.

The rest of the paper deals with (38).

The response of the liquid is detected through changes in the impedance of the NMR coil, which are proportional to (see Appendix B)

$$
\bar{\chi}=\left\langle h^{e+}\left|\widehat{\mathcal{G}}_{\omega}\right| h^{e+}\right\rangle,
$$

where $\widehat{\mathcal{G}}_{\omega}$ is the Green operator

$$
\left|m^{+}\right\rangle=\left(\omega_{M} / 4 \pi\right) \widehat{\mathcal{G}}_{\omega}\left|h^{e+}\right\rangle .
$$

Green operator may be expanded into an infinite sum in the eigenfrequencies $\omega_{\alpha}$ of the homogeneous equation corresponding to 38

$$
\widehat{\mathcal{H}}|\alpha\rangle=\omega|\alpha\rangle
$$

with the boundary condition

$$
\left.\widehat{n}_{i} \partial_{i}|\alpha\rangle\right|_{\partial}=0
$$

For a Hermitian Hamiltonian the eigenfrequencies $\omega_{\alpha}$ are real and the expansion is

$$
\widehat{\mathcal{G}}_{\omega}=\sum_{\alpha} \frac{|\alpha\rangle\langle\alpha|}{\omega_{\alpha}-\omega}
$$

The absorption spectrum in the Hermitian case consists of a series of $\delta$-peaks at $\omega=\omega_{\alpha}$. Indeed, writing real $\omega_{\alpha}$ in (45) as $\omega_{\alpha}+i 0$ we see that the imaginary (absorption) part of (41) is a weighted sum of $\delta$-functions

$$
-\pi \sum_{\alpha}\left|\left\langle\alpha \mid h^{e+}\right\rangle\right|^{2} \delta\left(\omega-\omega_{\alpha}\right)
$$

In the general case of a non-Hermitian Hamiltonian the expansion (45) should be revised. We postpone the appropriate discussion until Sec. V. Here it is enough to say that the numerators in the series (45) remain the same in the general case, but the eigenfrequencies $\omega_{\alpha}$ become complex, meaning that in general spectrum consists of Lorentzians.

The following important conclusion drawn on the basis of (45) also holds in the case of a non-Hermitian Hamiltonian. In a homogeneous $\left(\nabla \omega_{L}=0\right)$ external static fields 
$H^{e}$ and for ellipsoidal samples the Hamiltonian 40 has uniform solutions, so-called Kittel modes [14] with the frequencies

$$
\omega_{0}=\omega_{L}+\frac{\omega_{M}}{2}\left(1-3 \underline{n}_{z z}\right),
$$

where $\underline{n}_{z z}$ is the $\hat{z} \hat{z}$-component of the demagnetizing coefficient tensor $\underline{n}$.

For customary sample sizes the rf field $\mathbf{h}^{e}$ may be considered spatially uniform. Then from (46) it follows that it is impossible to excite a non-uniform mode by a homogeneous rf field $\mathbf{h}^{e}$, because then $\left\langle\alpha \mid h^{e+}\right\rangle \propto\langle\alpha \mid 0\rangle$, where $|0\rangle$ is the Kittel mode, and different modes are mutually orthogonal $\langle\alpha \mid 0\rangle=\delta_{\alpha 0}$.

We conclude that in order to couple to non-uniform eigenmodes the external static magnetic field should be inhomogeneous (see (19)) so that there would not exist a uniform eigenmode.

\section{FINITE-CYLINDRICAL CELL}

Study of the eigenstates of the Hamiltonian (40) in general is possible only numerically. This has already been done in Ref. |16], |17] neglecting the contribution of the dipolar field. The former work dealt with (40) in rectangular boxes, while the latter — in spherical containers.

In this paper we study the dipolar field effects due to the third term in (40) numerically in Section V. At the same time, in the case of $\omega_{M}=0$ the problem allows explicit analytical solution for some typical experimental conditions. Such solutions are of undeniable interest with them in hand we may use perturbation theory to calculate corrections to modes in the first order in $\omega_{M}$. So the two following sections are dedicated to such solutions and to the calculations of perturbational corrections respectively in the geometries of a finite cylinder and a sphere.

We start the consideration of finite cylinders from idealized one-dimensional geometry of a plane-parallel slab. Next we calculate the first order perturbations to the modes frequencies due to the finiteness of the cylinder.

\section{A. Slab}

In the absence of dissipation, when $C^{-1}=0$, the effective diffusion coefficient $D\left(1+i C^{-1}\right)$ is real and thus the Hamiltonian $\widehat{\mathcal{H}}(40)$ is Hermitian.

In the slab geometry the solution should be sought in the form

$$
m^{+}(\mathbf{r}) \propto e^{i \mathbf{k r}_{\perp}} m_{\mathbf{k}}^{+}(z),
$$

where $\mathbf{r}_{\perp}$ is the coordinate vector in the plane perpendicular to $\widehat{z}$. Nevertheless, as is clear from (41), only the solutions with $\mathbf{k}=0$ contribute to the observation signal if the $\mathrm{rf}$ field $\mathbf{h}^{e}$ is homogeneous.
The eigenfunctions $m_{0}^{+}(z)=\langle z \mid \alpha\rangle$ then are the combinations of the two Airy functions

$$
\langle z \mid \alpha\rangle=A \mathrm{Ai}(\arg )+B \operatorname{Bi}(\arg )
$$

where $\arg =\left(\omega_{\alpha}-\omega_{L}-z \nabla \omega_{L}\right) / \xi \nabla \omega_{L}$, and

$$
\xi=\sqrt[3]{D / \nabla \omega_{L}}
$$

is the characteristic wavelength. Its sign depends on the relative sign of $D$ and $\nabla \omega_{L}$ and thus on the sign of $\kappa$. In ${ }^{3} \mathrm{He}$ and in ${ }^{3} \mathrm{He}-{ }^{4} \mathrm{He}$ solutions with a concentration $x>$ $3.5 \%, \kappa$ is positive. We consider $\xi>0$ for definiteness.

The boundary conditions (44) on the two plane boundaries $\left.\partial_{z}|\alpha\rangle\right|_{z=0, L}=0$ determine the eigenfrequencies $\omega_{\alpha}$ and the ratio of the coefficients $B / A$. The remaining coefficient $A$ is determined from the normalization condition $\langle\alpha \mid \alpha\rangle=1$.

When $L \gg \xi$ the influence of the lower wall of the container is negligible and the modes are localized near the upper wall and decay exponentially into the bulk on distances $\sim \xi$. Then the eigenfunctions are just the Airy functions of the first kind - $\mathrm{Ai}$ and 48 becomes

$$
\langle z \mid \alpha\rangle \equiv\left\langle z \mid n_{z}\right\rangle=A \operatorname{Ai}\left(\frac{L-z}{\xi}+\alpha_{n_{z}}^{\prime}\right),
$$

where $\alpha_{n}^{\prime}<0$ is the $n$-th zero of the derivative of the Airy function $\mathrm{Ai}^{\prime}: \alpha_{1}^{\prime} \approx-1.02, \alpha_{2}^{\prime} \approx-3.25, \alpha_{3}^{\prime} \approx-4.82$, etc. The eigenfrequencies are

$$
\omega_{\alpha} \equiv \omega_{n_{z}}=\omega_{L}+L \nabla \omega_{L}+\alpha_{n_{z}}^{\prime} \xi \nabla \omega_{L}
$$

Inclusion of dissipation $C^{-1} \neq 0$ makes the diffusion coefficient complex. The Hamiltonian (40) then becomes non-Hermitian. The complete analysis of the spectra of a non-Hermitian Hamiltonian is possible only in the framework of the general formalism to be developed in Sec. V.

However for the moment it is sufficient to make the following statement. In the presence of dissipation $\left(C^{-1} \neq\right.$ $0) \xi$ in (49) becomes complex

$$
\xi \rightarrow \xi(1+i / C)^{1 / 3}
$$

and so do arguments of the eigenfunctions (48). Eigenfrequencies (50) also acquire imaginary parts due to $\xi$ entering the expression.

Thus the complex eigenfrequencies in the presence of dissipation can be easily obtained from the real ones in the absence by the substitution (51). This statement applies not only to (50) but to any spectrum of the Hamiltonian (40).

Let us now consider the effects of the dipolar field. We may utilize the results of Appendix A since a slab is infinite in the direction perpendicular to $\hat{z}$ and modes depend on only $z$. In such conditions the demagnetizing field is local

$$
\underline{\widehat{n}}_{z z}=n_{\mathrm{slab}}^{(z)}=1 \text {. }
$$


It is then obvious from (40) that the dipolar field produces no effect on the spin wave spectrum (50) apart from a uniform shift by

$$
\frac{1}{2} \omega_{M}\left(1-3 n_{\mathrm{slab}}^{(z)}\right)=-\omega_{M} .
$$

Such a shift does not distort the spectrum - it does not change neither the mutual positions of the modes nor their widths, from which the characteristics of the liquid are derived.

We rather aim at finding those distortions, so we proceed to a more relevant shape of a cylinder of a finite radius, which as well as all finite shapes as we will see does give such distortions.

\section{B. Finite cylinder}

Consider a finite cylinder with the base radius $R \gg \xi$ and height $L \gg \xi$ and a generatrix parallel to $\hat{z}$. We find the influence of the finiteness of a specimen on the magnitude of dipolar corrections to the spectrum in the first order of perturbation theory.

The first order perturbational corrections to the modes frequencies are the averages of the perturbation (dipolar) operator in the given eigenstate $\psi_{\alpha}(\mathbf{r})$

$$
\begin{aligned}
\delta_{\operatorname{dip}} \omega_{\alpha}=\frac{\omega_{M}}{2}(1 & -2 \int\left|\psi_{\alpha}(\mathbf{r})\right|^{2} \underline{\widehat{\underline{n}}}_{z z}[1] d^{3} \mathbf{r} \\
& \left.-\int \psi_{\alpha}^{*}(\mathbf{r}) \underline{\widehat{\underline{n}}}_{z z}\left[\psi_{\alpha}(\mathbf{r})\right] d^{3} \mathbf{r}\right) .
\end{aligned}
$$

In a finite cylinder the dipolar-free eigenfunction $|\alpha\rangle$ satisfying boundary conditions (44), written in cylindrical coordinates $z, \rho, \varphi$ is

$$
\psi_{n_{z} n_{\rho} m}=c_{n_{z} n_{\rho} m} \operatorname{Ai}\left(\frac{L-z}{\xi}+\alpha_{n_{z}}^{\prime}\right) J_{m}\left(\frac{\zeta_{n_{\rho} m}^{\prime}}{R} \rho\right) e^{i m \varphi},
$$

where $n_{z}, n_{\rho}=0,1,2, \ldots, \infty$ are respectively the longitudinal and radial quantum numbers and $m=-\infty, \ldots,+\infty$ is the azimuthal quantum number. $\zeta_{n_{\rho} m}^{\prime}$ is the $\left(n_{\rho}+1\right)$ th zero of the derivative $J_{m}^{\prime}$ of the Bessel function $J_{m}$, $c_{n_{z} n_{\rho} m}$ are the normalization coefficients.

From the general formula (41) it is not hard to see that only the modes with $n_{\rho}=m=0$ couple to the homogeneous rf field. Indeed, $\left|n_{z} 00\right\rangle$ is uniform in the plane perpendicular to $\hat{z}$. Therefore, integrals like $\left\langle n_{z} n_{\rho} m \mid h^{+e}\right\rangle$ are proportional to $\left\langle n_{\rho} m \mid 00\right\rangle=\delta_{n_{\rho} 0} \delta_{m 0}$.

Calculating (54) with (55) yields (see Appendix C)

$$
\begin{aligned}
\delta_{\text {dip }} \omega_{n_{z}}^{\text {cylinder }} & =\omega_{M}\left(-1+\frac{L}{\pi R} \log \frac{8 R}{e L}\right. \\
& \left.+\frac{\xi}{\pi R}\left(\Phi_{n_{z}} \log \frac{8 R}{e^{2} \Xi_{n_{z}} \xi}+\Psi_{n_{z}} \log \frac{e L}{\Theta_{n_{z}} \xi}\right)\right) .
\end{aligned}
$$

The first two terms not dependent on the mode number $n_{z}$ describe uniform shift of the spectrum, and the last two terms dependent on $n_{z}$ through the numerical constants $\Phi_{n_{z}}, \Psi_{n_{z}}, \Xi_{n_{z}}$ and $\Theta_{n_{z}}$, which are of the order of unity (see Table II in Appendix C), give the sought-for spectrum distortion.

We see that for finite $\xi / R$ the spectrum undergoes distortion proportional to the parameter

$$
\frac{2 \omega_{M}}{\pi \xi \nabla \omega_{L}} \frac{\xi}{R} \log \frac{\sqrt{R^{\Phi} L^{\Psi}}}{\xi^{\Phi+\Psi}}
$$

where $\omega_{M}=4 \pi \gamma M$ characterizes the magnetization density, $\nabla \omega_{L}$ is the gradient of the Larmor frequency, $R$ is the radius of the cylinder base, $L$ its height and $\xi$ is the wavelength (49) of an Airy-type standing spin wave. The quantity $\xi \nabla \omega_{L}$ gives the average distance between modes in the units of frequency. $\Phi$ and $\Psi$ are numbers of the order of unity.

The calculations of the dipolar field effects in the first order of perturbation theory allow us to estimate the maximum error due to the demagnetizing field in the determination of the transverse relaxation time $\tau$ from the spin wave spectra (see Appendix Q).

This error turns out to be of the order of the parameter (87), i.e. for the experiment $|10| \approx 4.2 \%$ for $M / M_{0} \sim 4$.

Thus, interpreting spectra according to the usual theory not taking into account the demagnetizing field induces an error in the derived value of the transverse relaxation time of the order of the parameter (87).

The term proportional to $\Phi_{n_{z}}$ comes from the demagnetizing field produced by the rotating part $\mathbf{m}$ of magnetization. While the term proportional to $\Psi_{n_{z}}$ is due to the spatial inhomogeneity of the demagnetizing field $-4 \pi \underline{\widehat{x}}[\mathbf{M}]$ produced by the initial static (homogeneous!) distribution of $\mathbf{M}(26)$ in a finite cylinder.

The values of these two terms are plotted in units of $\omega_{M}$ as functions of the mode number in Fig. 1 1 for $\xi / R=$ 0.015 and $L=2 R$. Apart from being greater, the term brought about by the spatial inhomogeneity of the static distribution of the dipolar field depends stronger on the mode number, thus resulting in bigger mutual shifts of the modes. So the main source of the spectrum distortion in a finite cylinder turns out to be the inhomogeneity of the static dipolar field.

In ellipsoids, in particular in a sphere, the demagnetizing field produced by the initial static distribution of $\mathbf{M}$ is homogeneous. And so there is only the distortion to the spectrum from the rotating part $\mathbf{m}$ of magnetization as we will see in the next section. This makes ellipsoidal shapes advantageous if the dipolar field effects are unfavorable.

To conclude, we found the corrections to the spin wave modes in a finite cylinder due to a weak demagnetizing field in perturbation theory. These corrections consist in shifting the spectrum as a whole, changing the relative distances between the modes and in narrowing down the modes. The two last are of interest for us since they deform the spectrum.

There are two contributions to the spectrum deformation - one from the static inhomogeneous demag- 


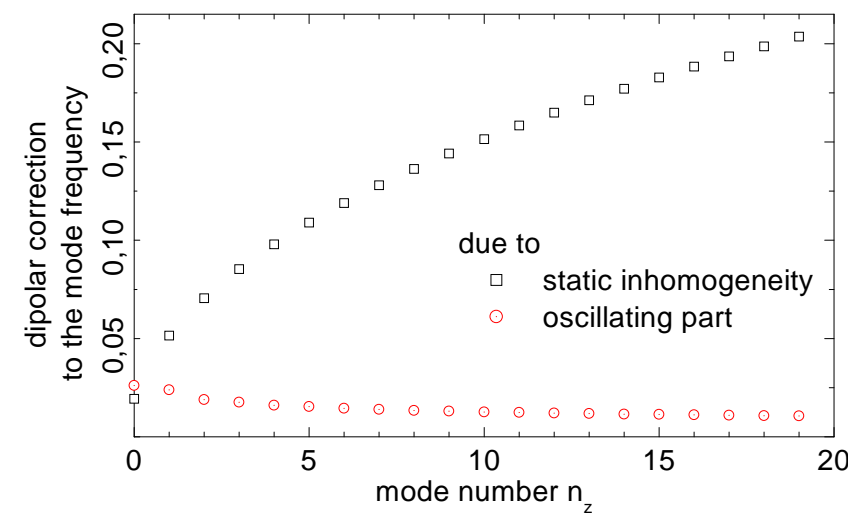

FIG. 1: Comparison of the two parts in the parameter of distortion of the spin-wave spectrum in a finite-cylindrical cel] brought about by the demagnetizing field (the two last terms of (56) in units of $\omega_{M}$ for $\xi / R=0.015$ and $L=2 R$ ). Th $\epsilon$ first part (third term in (56)) $(\bigcirc)$ is due to the demagnetizing field produced by the rotating part $\mathbf{m}$ of magnetization. The second part (the last term in $(56)$ ) ( $\square$ ) results from the inhomogeneity of the dipolar field produced by the initial static distribution $\mathbf{M}=\chi_{n} A H^{e} \hat{z}$ of magnetization in $\hat{\varepsilon}$ finite-cylindrical sample.

netizing field and the other from the rotating part of the magnetization. The first contribution exists only in nonellipsoidal samples, in which a homogeneous static magnetization produces inhomogeneous demagnetizing field.

\section{SPHERICAL CELL}

Though for a spherical container exact analytical solution in the absence of dipolar field turns to be impossible, one can obtain an explicit expression for several first modes in adiabatic approximation if the radius of the sphere $R \gg \xi$.

The prerequisites of adiabatic approximation might be best understood if one exploits the analogy with the Schrödinger equation for a particle moving in an external field. If the movement in one direction is somehow more restricted than in the others (geometrically or by an external field) it is a consequence of Heisenberg uncertainty relations that the movement in this direction will be faster. The slow enough movement in the other directions then will make up an adiabatic perturbation that is known not to change the state of the particle describing the fast motion.

As a result, the wave function can be combined as a multiplication of an envelope depending only on the unrestricted coordinates, and of the fast motion state depending on the unrestricted coordinates as on parameters.

This approach gives (see Appendix D) for the eigen-

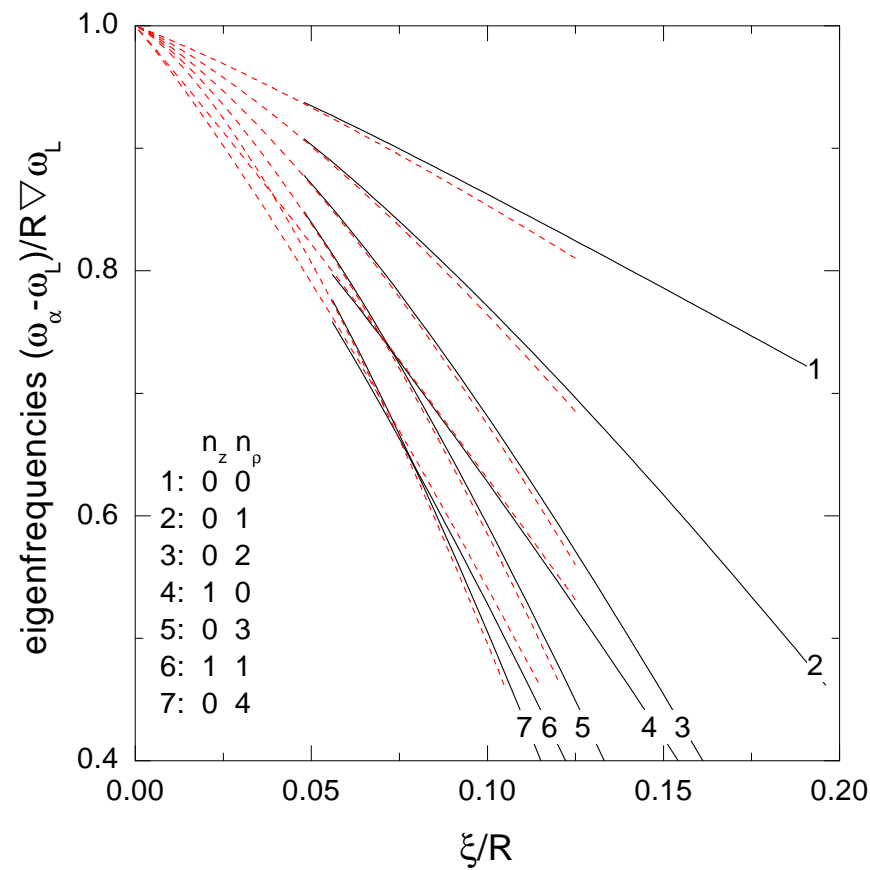

FIG. 2: Comparison of the first several spin wave modes frequencies in a spherical cavity obtained numerically (solid curves, 17]) and in the adiabatic approximation (dashed curves, Eq. (59)). The eigenfrequencies are plotted as functions of the ratio $\xi / R$ of the characteristic wavelength to the sphere radius.

frequencies

$$
\omega_{n_{z} n}=\omega_{L}+R \nabla \omega_{L}+\xi \nabla \omega_{L}\left[\alpha_{n_{z}}^{\prime}-\sqrt{2 \frac{\xi}{R}}\left(2 n_{\rho}+1\right)\right],
$$

where $\omega_{L}$ is the Larmor frequency in the center of the sphere, and $n_{z}, n_{\rho}=0,1,2, \ldots, \infty$ are the longitudinal and radial quantum numbers respectively. Since $\xi / R \ll$ $1 \sim \alpha_{n}^{\prime}$ we see that the lower-lying levels belong to $\alpha_{0}$ and therefore decay exponentially with diminishing $z$. Modes with $n_{z}=1$ will make a single oscillation before vanishing, modes with $n_{z}=2$ a double, etc.

Eq. (58) reduces to (50) in the limit $\xi / R \rightarrow 0$ as it must. Indeed, in both (58) and (50) there figures the Larmor frequency at the top of a sample $-z=L$ for (50) and $z=R$ for 58 ) and the second term in brackets in (58) tends to zero when $\xi / R \rightarrow 0$.

If we introduce dimensionless frequencies according to 117 $\omega_{\alpha}=\omega_{L}+R \nabla \omega_{L} f_{\alpha}$, we conclude that the observable eigenfrequencies for our problem are described in the adiabatic approximation by

$$
f_{\alpha}=1+\frac{\xi}{R}\left[\alpha_{n_{z}}^{\prime}-\sqrt{2 \frac{\xi}{R}}\left(2 n_{\rho}+1\right)\right] .
$$

The comparison between this formula and the results obtained numerically in Ref. $[17$ is shown in Fig. 2. In Ref. [17] the combination $\xi / R$ was designated as $\Delta$. 


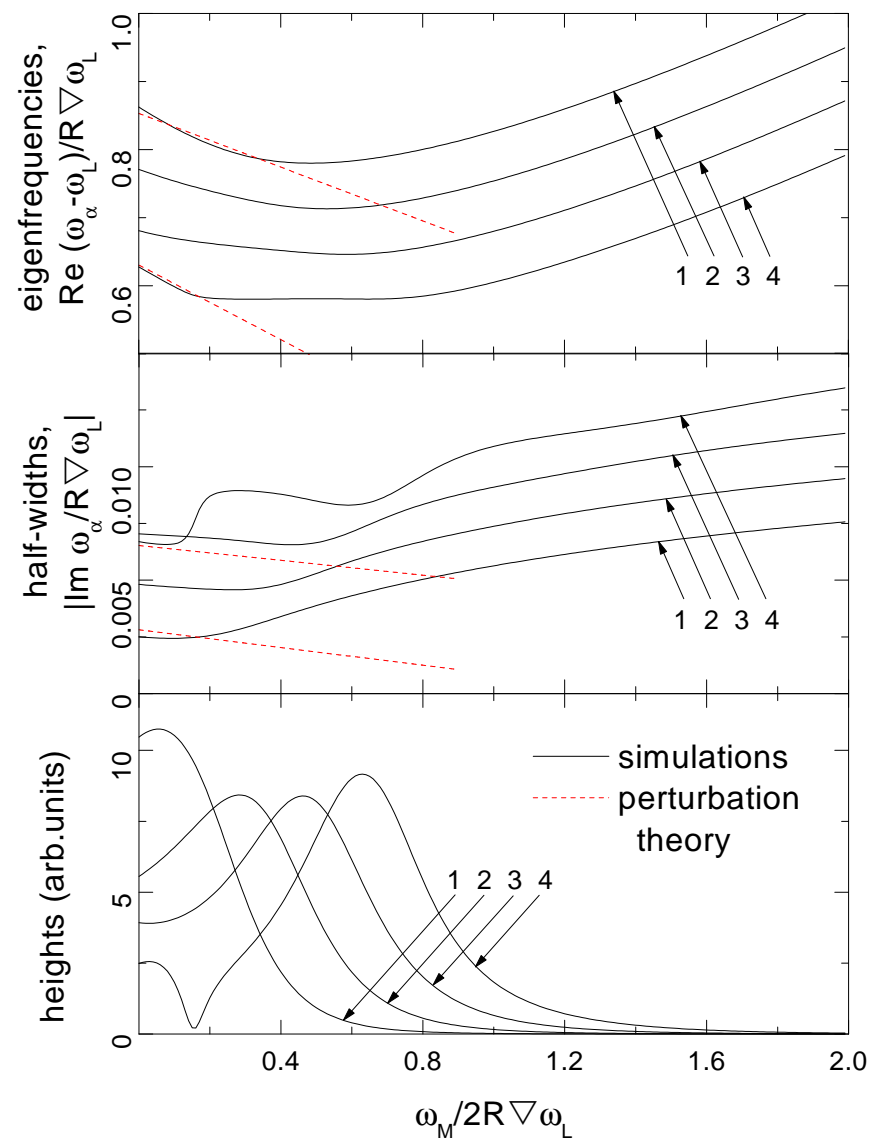

FIG. 3: The dependence of the frequencies, half-widths and heights of the first four modes (numbered in the same order as on Fig. 2) on the strength of the demagnetizing field $\omega_{M}$ (solid curves) obtained by numerical simulations in Section V. Input parameters are $\xi / R=0.1, C=20$. First order perturbational corrections (dashed lines) calculated in the text belong to the region $\omega_{M} / 2 R \nabla \omega_{L} \ll 1$. Perturbational calculations were done only for the modes with radial quantum numbers $n_{\rho}=0$, among the four depicted only the first and the fourth are of the type. Heights of the modes are impossible to calculate perturbationally.

Note that the numerical scheme developed in Ref. 17] requires more and more computational effort for $\xi$ tending to zero. The calculation time to get safe eigenfrequencies values grows. That is why the numerical curves are not shown in the vicinity of zero. Since we ourselves use a similar computational technique we put off more detailed discussion until Section V.

Contrariwise, the discrepancy between approximate and numerical curves at large $\xi$ is accounted for by inapplicability of adiabatics out of the region $\xi \ll R$.

So approximate and numerical methods complement each other. While numerics is the method of choice for relatively large $\xi / R$ when adiabatics breaks down, it requires increasingly larger basis to obtain reliable results for small $\xi / R$. In this region it is easier to calculate eigenfrequencies in adiabatic approximation.

Let us now look on dipolar field correction to the modes.

Quite analogously to the case of a finite cylinder, we obtain for the corrections to the modes (see Appendix D)

$$
\delta_{\mathrm{dip}} \omega_{\alpha}^{\text {sphere }}=\omega_{M}\left(-\frac{1}{3}+\frac{\Phi_{n_{z}} \sqrt{\pi}}{4} \sqrt[4]{\frac{\xi}{2 R}}\right) .
$$

And correspondingly for the corrections to the imaginary parts of the modes

$$
\delta_{\text {dip }} \Im \omega_{\alpha}^{\text {sphere }}=\frac{\omega_{M}}{12 C} \frac{\Phi_{n_{z}} \sqrt{\pi}}{4} \sqrt[4]{\frac{\xi}{2 R}} .
$$

Numerical constants $\Phi_{n_{z}}$ are the same as in (56).

The parameter determining the relative value of the dipolar field effects in a sphere is

$$
\frac{\sqrt{\pi} \omega_{M}}{4 \xi \nabla \omega_{L}} \sqrt[4]{\frac{\xi}{2 R}}
$$

In the next section we will solve the eigenvalue problem in a sphere in the presence of a dipolar field of an arbitrary strength. It is interesting to compare the results of numerical simulations with the first order perturbational corrections written above.

To this end the positions $\Re\left(\omega_{\alpha}-\omega_{L}\right)$ and half-widths $\Im \omega_{\alpha}$ of the first four modes obtained from both numerics and analytics are plotted on Fig. 3 for several $\omega_{M}$. Heights are plotted only as obtained numerically. As one can see from Fig. 3, perturbational approximation is satisfactory for the values of $\omega_{M} / 2 R \nabla \omega_{L}$ up to $\sim 0.1$, or, for values of (62) up to $\sim 0.4$. The small discrepancy between numerical and analytical results even in this region is accounted for by the restrictions of the adiabatic approximation used to fulfill analytical calculations.

The spectra themselves calculated numerically for several values of $\omega_{M}$ are shown on Fig. 4 . Modes weights are redistributing between adjacent modes with $\omega_{M}$ growing. Not only the weights but also half-widths and positions of the modes change.

\section{SPHERE. NUMERICAL CALCULATIONS}

The complexity of the Hamiltonian (40) does not allow finding exact eigenfunctions by analytical methods apart from using perturbation theory. Nevertheless it is always possible to solve equation (43) numerically. We chose a spherical container for numerical investigations.

Before proceeding to the description of the simulation scheme utilized, a formalism is to be established for solving an eigenvalue problem with a non-Hermitian Hamiltonian. Of interest for us is the generalization of the expansion (45) of the Green function into series over eigenfunctions. The following subsection is dedicated to the topic.

In the two remaining subsections we discuss respectively the technique and the results, other than already mentioned in the previous section, of the numerical simulations. 


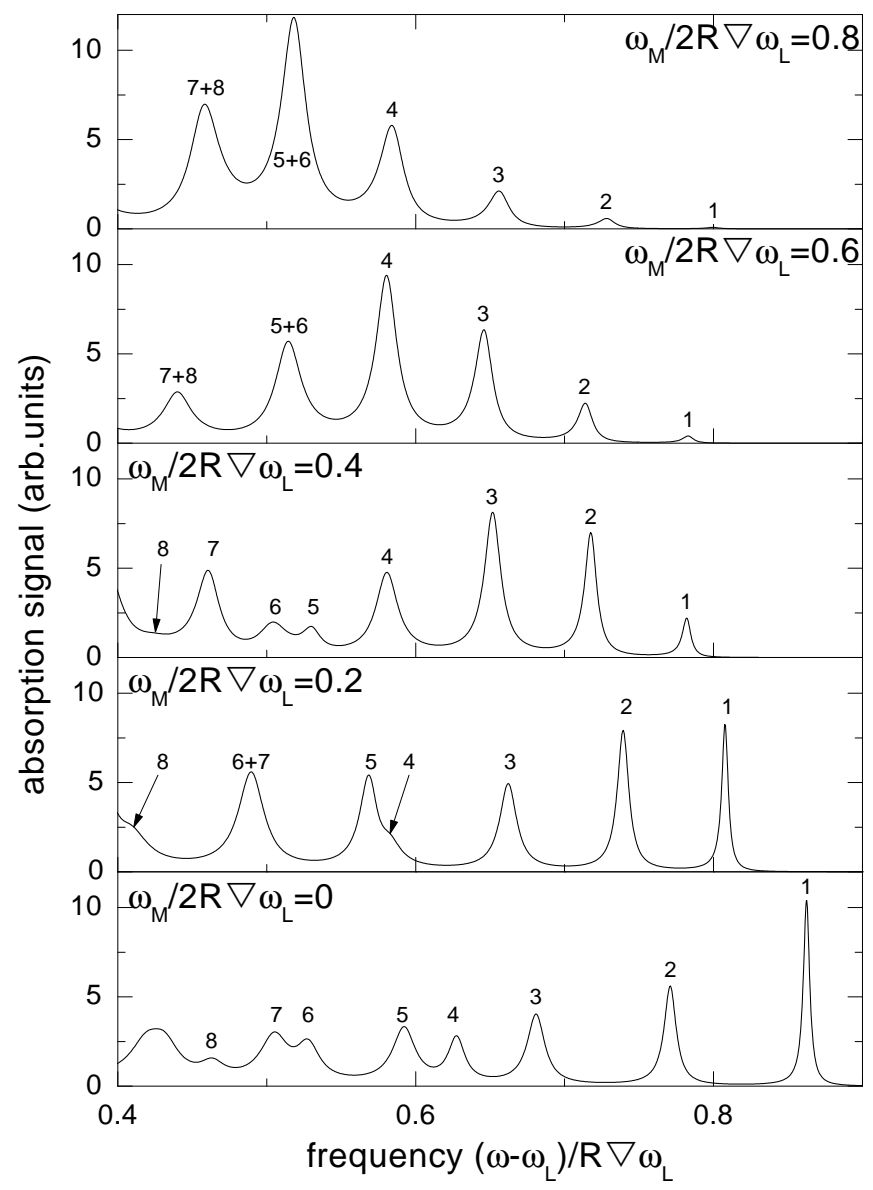

FIG. 4: Spin wave spectra obtained numerically in Section V for several $\omega_{M} / 2 R \nabla \omega_{L}<1$. Input parameters are $\xi / R=0.1$, $C=20$. Modes are numbered in the same order as on Fig. 2 .

\section{A. Green function of a non-Hermitian Hamiltonian}

In this subsection we derive an analogue of (45) for a non-Hermitian Hamiltonian $\widehat{\mathcal{H}} \neq \widehat{\mathcal{H}}^{+}$. For $\widehat{\mathcal{H}} \neq \widehat{\mathcal{H}}^{+}$ the set of eigenfunctions $|\alpha\rangle$ of $\widehat{\mathcal{H}}$ is not orthogonal. A second set of functions, viz., the set of eigenfunctions of the Hermitian conjugate operator $\widehat{\mathcal{H}}^{+}$, is to be introduced 24.

The eigenvalues of the operator $\widehat{\mathcal{H}}^{+}$are just the complex conjugates of the eigenvalues of $\widehat{\mathcal{H}}$. Indeed, the eigenvalues are found from a secular equation. And for the Hermitian conjugate operators these algebraic equations may be shown to be complex conjugate.

Thus the set of eigenfunctions of $\widehat{\mathcal{H}}^{+}$may always be numbered with the same index $\alpha$. To distinguish this set from $|\alpha\rangle$ we will denote it by $|\alpha\rangle$

$$
\begin{aligned}
\widehat{\mathcal{H}}|\alpha\rangle & =\omega_{\alpha}|\alpha\rangle, \\
\widehat{\mathcal{H}}^{+}|\alpha\rangle & =\omega_{\alpha}^{*}|\alpha\rangle .
\end{aligned}
$$

However, it should be borne in mind that $|\alpha\rangle$ like $|\alpha\rangle$ is an ordinary set of ket-vectors, which has the corresponding set of bra-vectors.
It turns out then that, notwithstanding that neither the set $|\alpha\rangle$ nor $|\alpha\rangle$ is orthogonal, there is orthogonality between the two sets. Indeed, following a conventional scheme of proving mutual orthogonality of eigenfunctions, we note that

$$
\langle\alpha|\widehat{\mathcal{H}}| \beta\rangle=\omega_{\beta}\langle\alpha \mid \beta\rangle
$$

On the other hand,

$$
\langle\alpha| \widehat{\mathcal{H}}=\left(\widehat{\mathcal{H}}^{+}|\alpha\rangle\right)^{+}=\left(\omega_{\alpha}^{*}|\alpha\rangle\right)^{+}=\omega_{\alpha}\langle\alpha| .
$$

Multiplying this by $|\beta\rangle$ and subtracting the previous result, we see that

$$
\left(\omega_{\alpha}-\omega_{\beta}\right)\langle\alpha \mid \beta\rangle=0 .
$$

Thus $|\beta\rangle$ and $|\alpha\rangle$ are orthogonal if $\alpha \neq \beta$. So it is said that the two sets $|\alpha\rangle$ and $|\alpha\rangle$ constitute a biorthogonal set of eigenfunctions.

The expansion of an arbitrary function into a convergent series is then possible

$$
|x\rangle=\sum_{\alpha}|\alpha\rangle\langle\alpha \mid x\rangle .
$$

Here the eigenfunctions are supposed to be normalized so that

$$
\langle\alpha \mid \beta\rangle=\delta_{\alpha \beta}
$$

Performing such an expansion for $\left|h^{+}\right\rangle$in (38), one obtains

$$
\widehat{\mathcal{G}}_{\omega}=\sum_{\alpha} \frac{|\alpha\rangle\langle\alpha|}{\omega_{\alpha}-\omega} .
$$

This is the sought-for generalization of (45). For a Hermitian Hamiltonian the two sets coincide $|\alpha\rangle=|\alpha\rangle$, and (69) reduces to (45).

Thus we see from (41) that the spectrum of a nonHermitian Hamiltonian consists of a set of Lorentzians at $\omega=\Re \omega_{\alpha}$ with half-widths $\Im \omega_{\alpha}$ each entering with a weight $\left\langle h^{e+} \mid \alpha\right\rangle\left\langle\alpha \mid h^{e+}\right\rangle$. If the rf field can be regarded as uniform on the scales of the sample, the relative weights of the Lorentzian peaks are

$$
\int\langle\mathbf{r} \mid \alpha\rangle d^{3} \mathbf{r} \int\left\langle\alpha \mid \mathbf{r}^{\prime}\right\rangle d^{3} \mathbf{r}^{\prime}
$$

In our particular case, the Hamiltonian 40 is symmetric $\widehat{\mathcal{H}}^{+}=\widehat{\mathcal{H}}^{*}$. This is trivial to see without the dipolar field term, but this term can also be shown to be (real) symmetric, since partial differential operator $\partial_{z}^{2}$ and the Green operator $\hat{\mathcal{G}}_{\infty}$ are both real symmetric and commutative $\partial_{z}^{2} \hat{\mathcal{G}}_{\infty}=\hat{\mathcal{G}}_{\infty} \partial_{z}^{2}$ (We remind that for an integral operator with a kernel $G\left(\mathbf{r}, \mathbf{r}^{\prime}\right)$ Hermitian conjugate has the kernel $\left.G^{*}\left(\mathbf{r}^{\prime}, \mathbf{r}\right)\right)$. Indeed, we write

$$
\partial_{z}^{2} \hat{\mathcal{G}}_{\infty} \mathbf{m}=\partial_{z}^{2} \int \frac{\mathbf{m}\left(\mathbf{r}^{\prime}\right)}{\left|\mathbf{r}-\mathbf{r}^{\prime}\right|} d^{3} \mathbf{r}^{\prime}=\int \mathbf{m}\left(\mathbf{r}^{\prime}\right) \partial_{z^{\prime}}^{2} \frac{1}{\left|\mathbf{r}-\mathbf{r}^{\prime}\right|} d^{3} \mathbf{r}^{\prime}
$$


Taking the integral two times by parts and taking into account that $\mathbf{m}\left(\mathbf{r}^{\prime}\right) /\left|\mathbf{r}-\mathbf{r}^{\prime}\right| \rightarrow 0$ at $z^{\prime} \rightarrow \pm \infty$, we get

$$
\int \frac{\partial_{z^{\prime}}^{2} \mathbf{m}\left(\mathbf{r}^{\prime}\right)}{\left|\mathbf{r}-\mathbf{r}^{\prime}\right|} d^{3} \mathbf{r}^{\prime}=\hat{\mathcal{G}}_{\infty} \partial_{z}^{2} \mathbf{m}
$$

Therefore from the definition (64) we immediately conclude that in the case of a symmetric Hamiltonian $\widehat{\mathcal{H}}^{+}=\widehat{\mathcal{H}}^{*}$ the two sets of eigenfunctions are related through $\langle\mathbf{r} \mid \alpha\rangle=\langle\mathbf{r} \mid \alpha\rangle^{*} \equiv\langle\alpha \mid \mathbf{r}\rangle$. Expression (70) for the relative weights of the modes simplifies then to

$$
\left(\int\langle\mathbf{r} \mid \alpha\rangle d^{3} \mathbf{r}\right)^{2}
$$

Note that the (complex) value itself of the integral is squared, not its absolute value, as it would be should the Hamiltonian be Hermitian. We will use the expression (73) for the modes weights in numerical calculations of the spectra.

\section{B. Numerical approach}

One of the methods for solving a spectral SturmLiouville problem (conceptually, perhaps, the simplest) consists in its finite-dimensional approximation. Formally, we then are left with standard algebraic spectral problem. For the solution of the latter one can implement one of ready safe well-established algorithms. However, one must be cautious with the dimension of approximation.

One of the possible discretization techniques is to find eigenfunctions in the representation of some complete orthonormal set of functions when the Hamiltonian 40) would become a matrix. Such a scheme was developed in application to spin waves in Ref. [16], 17.

A handy orthonormal set to choose is that of eigenfunctions of the Laplace operator satisfying the boundary conditions for the geometry in question

$$
\begin{aligned}
{\left[\partial^{2}+k_{\mu}^{2}\right]|\mu\rangle } & =0 \\
\left.\widehat{n}_{i} \partial_{i}|\mu\rangle\right|_{\partial} & =0
\end{aligned}
$$

Here $\mu$ stands for a complete set of indices needed to describe a state, $k_{\mu}$ are waveconstants. In the case of such a choice of the set the first term of the Hamiltonian (40) becomes trivial and the boundary conditions are met automatically.

The eigenfunctions $|\alpha\rangle,|\alpha\rangle$ of the operators $\widehat{\mathcal{H}}, \widehat{\mathcal{H}}^{+}$ then take the form

$$
|\alpha\rangle=\sum_{\mu}|\mu\rangle\langle\mu \mid \alpha\rangle, \quad|\alpha\rangle=\sum_{\nu}|\nu\rangle\langle\nu \mid \alpha\rangle .
$$

where the coefficients of expansion $\langle\mu \mid \alpha\rangle$ and $\langle\nu \mid \alpha\rangle$ are found numerically as right and left eigenvectors of the matrix form of the Hamiltonian (40) corresponding to eigenfrequencies $\omega_{\alpha}$

$$
\begin{aligned}
\sum_{\nu} \mathcal{H}_{\mu \nu}\langle\nu \mid \alpha\rangle & =\omega_{\alpha}\langle\mu \mid \alpha\rangle, \\
\sum_{\nu}\langle\alpha \mid \nu\rangle \mathcal{H}_{\nu \mu} & =\omega_{\alpha}\langle\alpha \mid \mu\rangle,
\end{aligned}
$$

where $\mathcal{H}_{\mu \nu}=\langle\mu|\widehat{\mathcal{H}}| \nu\rangle$.

For a sphere $\mu$ denotes the set $n, l, m$ of the radial, polar and azimuthal quantum numbers: $n, l=0,1,2$, $\ldots, m=-l,-l+1, \ldots, l-1, l$. The corresponding basis is

$$
\langle\mathbf{r} \mid \mu\rangle=\langle\mathbf{r} \mid n l m\rangle=c_{n l} j_{l}\left(k_{n l} r\right) Y_{l}^{m}(\widehat{\mathbf{r}})
$$

where $j_{l}$ is the spherical Bessel function, $Y_{l}^{m}$ is the spherical harmonic, and renormalization coefficients $c_{n l}$ are defined according to

$$
c_{n l} c_{n^{\prime} l} \int_{0}^{R} j_{l}\left(k_{n l} r\right) j_{l}\left(k_{n^{\prime} l} r\right) r^{2} d r=\delta_{n n^{\prime}} .
$$

The waveconstants $k_{n l}$ depend on the boundary condition. That of $(75)$ requires that $k_{n l} R$ be the $(n+1)$-th zero of the derivative $\partial_{r} j_{l}(r)$ of the spherical Bessel function.

Since we are interested only in axisymmetric modes, which couple to a homogeneous rf field, we may simplify the formulas by working with a sub-basis $|n l 0\rangle$.

Infinite indexing $n, l$ is to be truncated at some finite values for numerical computation. Maximum values of $n_{\max }, l_{\max }$ are restricted by computational tractability of resulting matrices.

On the other hand, justification for such a truncation is that coefficients $\langle n l \mid \alpha\rangle$ tend to zero for large $n, l$ because of the oscillating character of $j_{l}$. We expect $\langle n l \mid \alpha\rangle$ close to zero when the characteristic scale $\xi$ of the function $\langle\mathbf{r} \mid \alpha\rangle$ becomes greater then the period $\sim R / n$ of the oscillations of the basis function $j_{l}$. Empirically, $n_{\max }=10$ is already quite good for customary $\xi / R \sim 0.1$. Note that the change in $j_{l}\left(k_{n l} r\right)$ with increasing $l$ is much less dramatic. So more $l$ 's are to be retained in the sub-basis. We used $l_{\max }=51$. Further increase of $n_{\max }, l_{\max }$ proved to have no apparent effect on the spectra for $\xi / R \sim 0.1$.

However, for $\xi / R \rightarrow 0$ more and more sub-basis functions should be kept, which leads to rapid slowing down of the computations. In this limit adiabatic approximation (see Sec. IV) gives safer results.

It is convenient to seek for the eigenfrequencies $\omega_{\alpha}$ in the form $\omega_{L}+R \nabla \omega_{L} f_{\alpha}$. The Hamiltonian for the matrix equation on $f_{\alpha}$

$$
\begin{aligned}
\left\langle n l\left|\widehat{\mathcal{H}}_{f}\right| n^{\prime} l^{\prime}\right\rangle & =-\left(\frac{\xi}{R}\right)^{3}\left(1+\frac{i}{C}\right)\left(k_{n l} R\right)^{2} \delta_{n n^{\prime}} \delta_{l l^{\prime}} \\
& +\left\langle n l\left|\frac{z}{R}\right| n^{\prime} l^{\prime}\right\rangle \\
& +\frac{\omega_{M}}{2 R \nabla \omega_{L}}\left\langle n l\left|\frac{1}{3}-\underline{\widehat{n}}_{z z}\right| n^{\prime} l^{\prime}\right\rangle
\end{aligned}
$$

comprises three parameters - $\xi / R$, the ratio $\omega_{M} / 2 R \nabla \omega_{L}$ of $\omega_{M}$ to the total field gradient over 
the sample and the regime parameter $C$. Here we have used $1-2 n_{\text {sphere }}^{(z)}=\frac{1}{3}$.

The matrix elements of $z / R$ and of $\widehat{\underline{\underline{ }}}_{z z}$ by integrating over the solid angle with appropriate spherical functions reduce to integrals over the radial coordinate $r$.

The matrix elements of $z / R$ were written in [17], the integrals arising should be calculated numerically. The rather lengthy calculations of the matrix elements of $\underline{\underline{n}}_{z z}$ were separated into Appendix E.

We cite here only the results. The matrix elements of $z / R=r \cos \theta / R$, where $\theta$ is the spherical polar angle are non-zero only if $l^{\prime}=l \pm 1$

$$
\begin{aligned}
\langle n l| & z / R\left|n^{\prime}, l^{\prime}=l \pm 1\right\rangle \\
& =c_{\lambda}^{0} c_{n l} c_{n^{\prime} l^{\prime}} \int_{0}^{R} j_{l}\left(k_{n l} r\right) j_{l^{\prime}}\left(k_{n^{\prime} l^{\prime}} r\right) r^{3} d r / R .
\end{aligned}
$$

Here $\lambda$ is the greater of $l, l^{\prime}$ and

$$
c_{\lambda}^{0}=\lambda / \sqrt{4 \lambda^{2}-1} .
$$

The matrix elements of $\underline{\hat{\underline{n}}}_{z z}$ are non-zero only if $l^{\prime}=$ $\{l, l \pm 2\}$ (see $(\mathrm{E} 28)$ in Appendix $\mathbb{E}$ )

$$
\begin{aligned}
& \left\langle n \quad l\left|\frac{1}{3}-\underline{\widehat{n}}_{z z}\right| n^{\prime} l\right\rangle=\delta_{n n^{\prime}}\left[\frac{1}{3}-\left(c_{l}^{0}\right)^{2}-\left(c_{l+1}^{0}\right)^{2}\right] \\
& \left\langle n \quad l\left|\frac{1}{3}-\underline{\widehat{n}}_{z z}\right| n^{\prime}, l^{\prime}=l \pm 2\right\rangle=c_{\lambda}^{0} c_{\lambda-1}^{0} c_{n l} c_{n^{\prime} l^{\prime}} R^{2} \\
& \times \frac{k_{n l} j_{l+1}\left(k_{n l} R\right) j_{l}\left(k_{n^{\prime} l^{\prime}} R\right)-k_{n^{\prime} l^{\prime}} j_{l+1}\left(k_{n^{\prime} l^{\prime}} R\right) j_{l}\left(k_{n l} R\right)}{k_{n l}^{2}-k_{n^{\prime} l^{\prime}}^{2}}
\end{aligned}
$$

It may be verified that the matrix $\left\langle n l\left|\widehat{\mathcal{H}}_{f}\right| n^{\prime} l^{\prime}\right\rangle$ is indeed symmetric.

The algebraic eigenvalue problem for (81) with (82) was solved using standard subroutine from the Linear Algebra Package LAPACK. Eigenvectors were then normalized and the left and right eigenvectors used to find the modes weights.

In calculating modes weights using (77), (78) we note that $\langle\mathbf{r} \mid 000\rangle=1 / \sqrt{V}$, where $V=4 \pi R^{3} / 3$ is the sphere volume. Thus

$$
\int\langle\mathbf{r} \mid n l m\rangle d^{3} \mathbf{r}=\sqrt{V}\langle 000 \mid n l m\rangle=\sqrt{V} \delta_{n 0} \delta_{l 0} \delta_{m 0} .
$$

The general formula $(70)$ in this case reduces to

$$
V\langle 000 \mid \alpha\rangle\langle\alpha \mid 000\rangle .
$$

\section{Results of simulations}

Numerical results in the absence of the dipolar field were obtained in Ref. |17|. When $\omega_{M}=0$ there remain only two parameters in the problem - the ratio $\xi / R$ of the characteristic wavelength to the sphere radius and the regime parameter $C$ which determines half-widths of the modes. The dependence of the spin wave spectrum on $\xi / R$ in the limit of weak demagnetizing field was plotted

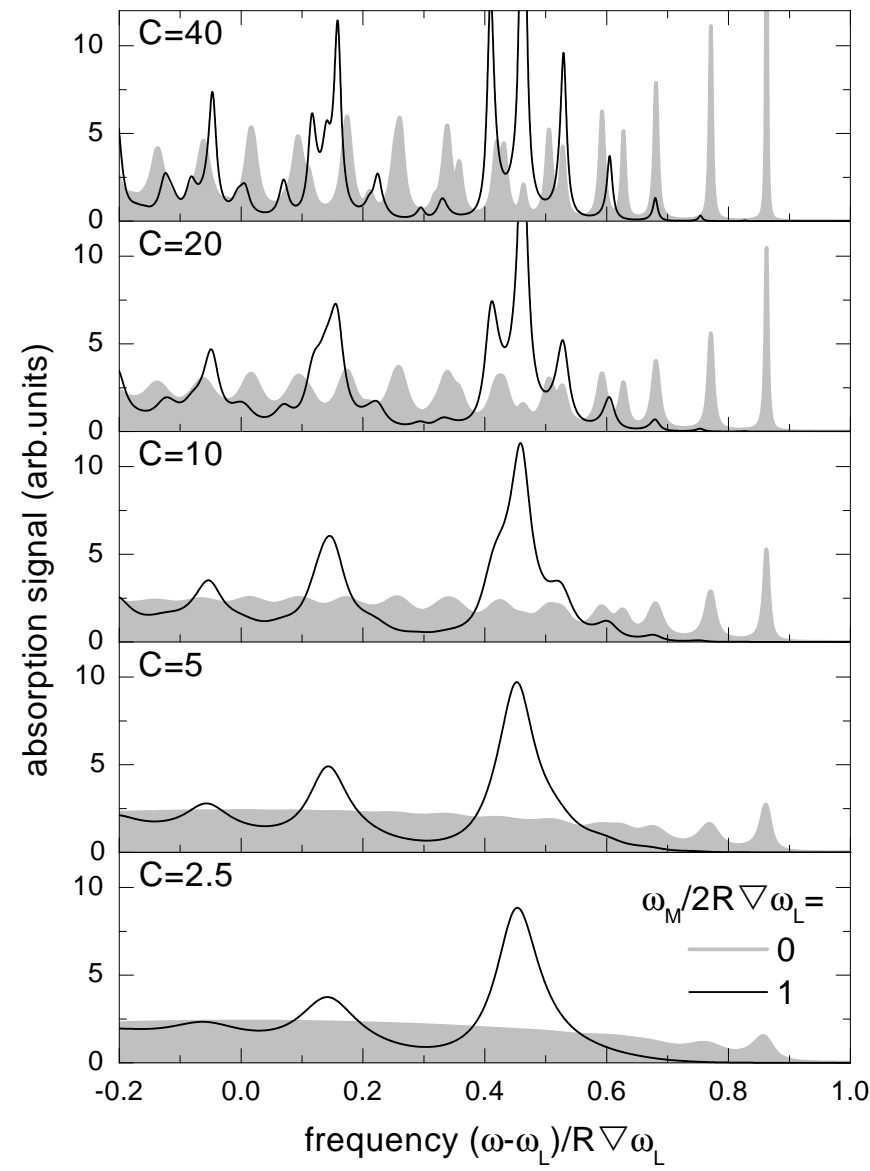

FIG. 5: Absorption signals of a polarized Fermi liquid in a spherical container for several regime parameters $C$ in neglect of the dipolar field effects $\omega_{M}=0$ (grey filled curves) and for a dipolar field of an intermediate strength $\omega_{M} / 2 R \nabla \omega_{L}=1$ (solid curves). The ratio $\xi / R$ was put 0.1 .

in Fig. 2 to compare adiabatic approximation with simulations. A typical absorption signal in the absence of the dipolar field is depicted as grey filled curves in Fig. 固 for several $C$. Decrease in $C$ results in modes broadening without changing their positions.

When $\omega_{M}$ is small enough the dipolar field constitutes a perturbation to conventional Silin spin waves. It is this regime which was studied perturbationally in Sec. IV.2.

In this subsection we shortly consider other results of numerical calculations, viz., regimes of intermediate and strong demagnetizing fields. Although these regimes were not realized so far in Fermi liquids (see Table I), one cannot leave out what is to be expected.

As $\omega_{M}$ increases the spin wave spectrum undergoes crossover from Silin type for small demagnetizing fields $\left(\omega_{M} / 2 R \nabla \omega_{L}<1\right)$ to magnetostatic type for large demagnetizing fields $\left(\omega_{M} / 2 R \nabla \omega_{L}>1\right)$. On a gross scale this transition is represented in Fig. 6 for $\xi / R=0.1$ and $C=20$ (grey filled curves) and $C=5$ (solid curves). Modes weights change so that adjacent Silin modes group into fewer magnetostatic modes. These separate at even 
larger $\omega_{M}$ until a uniform (Kittel) mode singles out at extremely large $\omega_{M}$.

This latter mode is the only one to remain because we chose a uniform radio-frequency field for the response of the system. And a non-uniform rf field is required to couple to non-uniform magnetostatic modes since the influence of external field gradient $\nabla \omega_{L}$ is negligible for large $\omega_{M}$.

The behavior described is not altered by larger dissipation (smaller $C$ ) other than Silin modes grouping becomes more pronounced (see solid curves on Fig. 6).

The dependence on $C$ of a spectrum for a demagnetizing field of an intermediate strength $\left(\omega_{M} / 2 R \nabla \omega_{L}=1\right)$ is plotted in Fig. 5 as solid curves. Pronounced adjacent modes for larger $C$ merge into magnetostatic conglomerates with no distinction for smaller $C$. No apparent relation, especially for smaller $C$, can be seen with the spectrum in the absence of the demagnetizing field.

\section{DISCUSSIONS}

\section{A. The method}

We studied the influence of the dipolar (or demagnetizing) field on the spectrum of linear standing spin waves in a polarized Fermi liquid in a finite container.

A somewhat resembling problem was studied in the 70s in ferrimagnets. deWames and Wolfram [28] considered not the Larmor-precession case (11), but the true LandauLifshitz equations of inhomogeneous magnetization dynamics that contain an additional exchange torque term depending on the Laplacian of the magnetization. This term is similar to that arising from the Leggett equations (see Eq. (30)).

The situation in ferrimagnets and in polarized Fermiliquids belong however to different limiting cases. In ferrimagnets the dipolar field effects - magnetostatic waves - are pronounced and exchange is a perturbation. In a paramagnetic Fermi liquid under conventional experimental conditions, on the contrary, it is the dipolar field whose effect is smaller.

There is another obstacle of transplanting the approach of deWames and Wolfram to our needs. In order to accommodate all the inhomogeneities - both from the Landau-Lifshitz equations and from the Maxwell equations (8) - the resulting differential equation for magnetostatic potential is of the sixth order in spatial derivatives. Though it was manageable in a quasi-1D situation (ferrimagnetic substance yttrium iron garnet YIG is grown as thin films), it becomes virtually intractable in any, even the simplest, 3D geometry.

An approach to include dipolar field into magnetization dynamics analogous to ours was used by Deville et al. 229 in application to solid bcc ${ }^{3} \mathrm{He}$. In this material the dipolar field gives rise to multiple spin echos at times $n \tau$ following two isolated $\mathrm{rf}$ pulses at times 0 and $\tau$. Ref. [29] explained this phenomenon quantitatively using

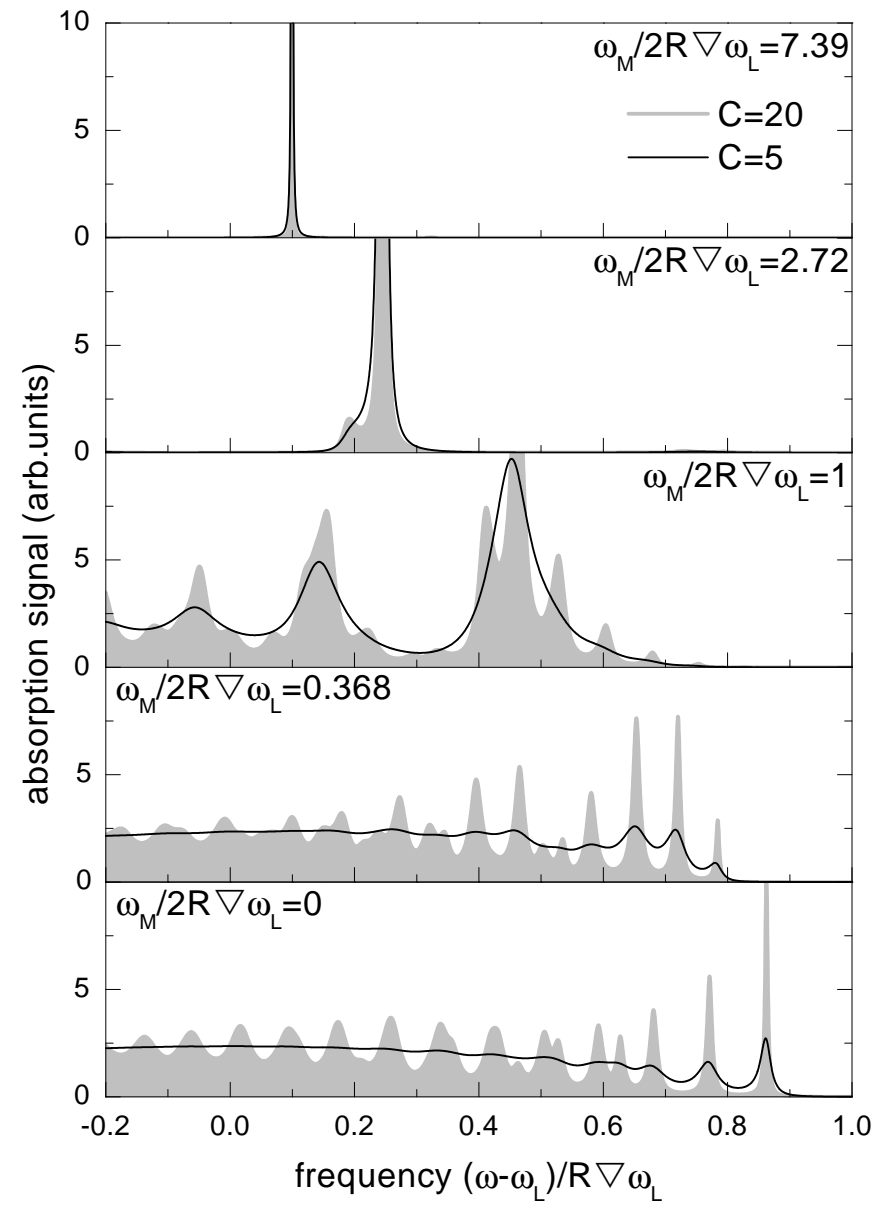

FIG. 6: From bottom to top - evolution of the spin waves spectrum of a polarized Fermi liquid at a crossover from weak to strong demagnetizing field. Several adjacent Silin modes form a group which evolves further as a magnetostatic whole. The distance between magnetostatic modes grows with $\omega_{M}$ until we are left with the sole mode in which magnetization in the whole sample oscillates uniformly. Input parameters were $\xi / R=0.1, C=20$ (grey filled curves) and $C=5$ (solid curves).

a simplified local approximation for the dipolar field valid for a slab infinite in the directions perpendicular to the external field (although this restriction was not properly emphasized in the paper). Later Fomin and Vermeulen [30| utilized the form of the dipolar field term of Ref. [29] to study dipolar corrections to a two-domain coherently precessing structure.

As for linear spin waves, in this local approximation neither the relative positions of the modes nor their halfwidths and heights change, and the spectrum only shifts as a whole. The demagnetizing field is by definition shape-dependent and it is erroneous to use the local approximation a priori. 
TABLE I: Comparison of the conditions of different spin-wave experiments [10], [16], |17, |18 in pure ${ }^{3} \mathrm{He}$ and in solutions of ${ }^{3} \mathrm{He}$ in ${ }^{4} \mathrm{He}$. From left to right. i) The concentration $x$ of ${ }^{3} \mathrm{He}$ atoms in mixtures (for pure ${ }^{3} \mathrm{He}$ not given). ii) The static

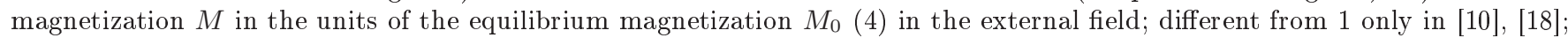
see the text for more explanation. iii) The Larmor frequency $\omega_{L} / 2 \pi$, where $\omega_{L}=\gamma H^{e}$. iv) The gradient $\nabla H^{e}=\nabla \omega_{L} / \gamma$ of external field; v) The mean distance between modes $\xi \nabla \omega_{L}$ in the units of frequency, $\xi$ being the characteristic spatial distance (49) - the wavelength of Airy type spin wave. vi) $\omega_{M}=4 \pi \gamma M-$ a characteristic of the static demagnetizing field. vii) The radius $R$, for a box this is half-size, for factually used cylinders it is also roughly the half-height. viii) - x) The three parameters entering the Hamiltonian (81) used for simulations of the eigenvalue problem in a spherical cell, $(\xi / R)^{3}$ and $\omega_{M} / 2 R \nabla \omega_{L}$ give the relative importance correspondingly of the exchange molecular field and of the demagnetizing field to the gradient of the external field; in a box these parameters lack strict meaning and are presented here in parentheses only for an estimate; $C$ is the regime parameter (5) at the temperature shown for each experiment in the left column. xi) The dipolar parameter (87) (cylinder and box) or (88) (sphere) (more exactly, their more accurate equivalents (56) and (60) respectively); if it is much smaller than unity it signifies that perturbation theory can be applied at the conditions of the experiment. The input parameters for the calculations come from various sources [19].

\begin{tabular}{|c|c|c|c|c|c|c|c|c|c|c|c|}
\hline $\begin{array}{l}\text { parameters } \\
\text { and units }\end{array}$ & $\begin{array}{c}\text { concen- } \\
\text { tration } \\
x \\
10^{-3} \\
\end{array}$ & $\begin{array}{l}\text { magneti- } \\
\text { zation } \\
\frac{M}{M_{0}}\end{array}$ & $\begin{array}{c}\text { Larmor } \\
\text { frequency } \\
\frac{\omega_{L}}{2 \pi}, \\
\mathrm{MHz}\end{array}$ & $\begin{array}{c}\text { field } \\
\text { gradient } \\
\nabla H^{e}, \\
\mathrm{G} / \mathrm{sm}\end{array}$ & $\begin{array}{c}\text { modes } \\
\text { distance } \\
\frac{\xi \nabla \omega_{L}}{2 \pi} \\
\mathrm{Hz}\end{array}$ & $\begin{array}{l}\frac{\omega_{M}}{2 \pi} \\
\mathrm{Hz}_{\mathrm{z}}\end{array}$ & $\begin{array}{r}R, \\
\mathrm{~mm}\end{array}$ & $\begin{aligned} & \frac{\xi}{R} \\
& \times 10^{-3} \\
&\end{aligned}$ & $C$ & $\begin{array}{l}\frac{\omega_{M}}{2 R \nabla \omega_{L}} \\
\times 10^{-3}\end{array}$ & $\begin{array}{c}\text { dipolar } \\
\text { parameter } \\
(87) \text { or }(88) \\
\times 10^{-3}\end{array}$ \\
\hline \multirow{3}{*}{$\begin{array}{l}\text { pure }{ }^{3} \mathrm{He} \text { in a } \\
\text { rectangular box, } \\
16, T=1 \mathrm{mK}\end{array}$} & & 1 & 1 & 2 & 122 & 0.48 & & $(189)$ & 3.7 & $(0.37)$ & 1.17 \\
\hline & - & 1 & 2 & 2 & 97 & 0.97 & 1 & $(150)$ & 7.3 & $(0.74)$ & 2.5 \\
\hline & & 1 & 4 & 2 & 77 & 1.93 & & (119) & 14.6 & $(1.49)$ & 5.5 \\
\hline \multirow{2}{*}{$\begin{array}{c}{ }^{3} \mathrm{He}^{-4} \mathrm{He} \text { in a sphere, } \\
\text { |17, } T=20 \mathrm{mK}\end{array}$} & 1.82 & 1 & 258 & 3.62 & 60 & 9.2 & 0.4 & 133 & 2.5 & 10.1 & 38 \\
\hline & 0.63 & 1 & 258 & 2.01 & 30 & 3.9 & 0.6 & 77 & 3 & 4.95 & 28 \\
\hline${ }^{3} \mathrm{He}-{ }^{4} \mathrm{He}$ in $\mathrm{a}$ & & 1 & & & 78.4 & 23.5 & & 121 & 9.7 & 18.1 & 73 \\
\hline hemisphere, 18, & & 2 & & & 36.2 & 47.0 & & 96 & 19 & 36.3 & 173 \\
\hline \multirow[t]{2}{*}{$T=20 \mathrm{mK}$} & 93 & 3 & 341 & 4 & 54.4 & 70.6 & 0.5 & 84 & 29 & 54.4 & 288 \\
\hline & & 4 & & & 49.4 & 94.1 & & 76 & 39 & 72.5 & 413 \\
\hline pure ${ }^{3} \mathrm{He}$ in & & 1 & & & 21.9 & 241 & & 6.6 & 2.3 & 36 & 252 \\
\hline a finite & & 2 & & & 17.4 & 482 & & 5.2 & 4.5 & 73 & 532 \\
\hline cylinder, 18], & - & 3 & 312 & 5.1 & 15.2 & 723 & 2.0 & 4.6 & 6.8 & 109 & 809 \\
\hline \multirow[t]{2}{*}{$T=20 \mathrm{mK}$} & & 4 & & & 13.8 & 963 & & 4.2 & 9.1 & 146 & 1099 \\
\hline & & 5 & & & 12.8 & 1204 & & 3.9 & 11 & 182 & 1385 \\
\hline${ }^{3} \mathrm{He}-{ }^{4} \mathrm{He}$ in & & 1 & & & 150 & 23.5 & & 22 & 9.6 & 1.7 & 9.6 \\
\hline a finite & & 2 & & & 119 & 47.0 & & 17 & 19 & 3.4 & 20 \\
\hline cylinder, $[10]$, & 93 & 3 & 341 & 10.6 & 104 & 70.6 & 2.0 & 15 & 29 & 5.1 & 31 \\
\hline$T=20 \mathrm{mK}$ & & 4 & & & 95 & 94.6 & & 14 & 39 & 6.8 & 42 \\
\hline
\end{tabular}

\section{B. Main results}

The strength of the demagnetizing field is proportional to the polarization of the liquid. So in conventional weakly-polarized liquids the dipolar field can be neglected, while for strong enough polarizations its effects overwhelm usual Fermi liquid exchange field spin dynamics.

In particular, the influence of the demagnetizing field on the spectrum of standing spin waves in an (infi-
Because of its long-range character, the dipolar interaction introduces an additional non-local term into the equations of the spin dynamics. This term is an integrodifferential operator on the magnetization, wherein the integration is taken over the volume of the liquid. As a consequence the corrections to the spin dynamics depend strongly on the shape of the container.

nite) slab reduces for an arbitrary strength of the dipolar field simply to uniform shift of all the modes by 
$-\omega_{M}=-4 \pi \gamma M$, where $M$ is the static magnetization of the liquid in an external field, $\gamma$ - gyromagnetic ratio.

In a finite volume of a liquid strengthening of the demagnetizing field results in the crossover from the Silin type spin wave regime to the regime of magnetostatic waves. In this latter the mechanism of the forming of the standing spin waves has nothing to do with the exchange physics of Fermi liquids. Magnetostatic spectra are in no way specific to the Fermi liquid and so contain no information on its parameters.

For intermediate polarizations the demagnetizing field in a finite volume of a liquid leads to the corrections to Silin type spin wave spectra, both shifting the spectra uniformly and also changing the distances between the modes and the modes widths. These changes of the second type which distort the spectra are undesirable from the point of view of deriving parameters of the liquid from the spectra.

We have seen that the parameter determining the influence of the demagnetizing field on the spectra of spin waves in a finite cylinder in the first order of perturbation theory is

$$
\frac{2 \omega_{M}}{\pi \xi \nabla \omega_{L}} \frac{\xi}{R} \log \frac{\sqrt{R^{\Phi} L^{\Psi}}}{\xi^{\Phi+\Psi}},
$$

where $\omega_{M}=4 \pi \gamma M$ characterizes the magnetization density, $\nabla \omega_{L}$ is the gradient of the Larmor frequency, $R$ is the radius of the cylinder base, $L$ its height and $\xi$ is the wavelength (49) of an Airy-type standing spin wave. The quantity $\xi \nabla \omega_{L}$ gives the average distance between modes in the units of frequency. $\Phi$ and $\Psi$ are numbers of the order of unity.

For a sphere, an analogous parameter was

$$
\frac{\sqrt{\pi} \omega_{M}}{4 \xi \nabla \omega_{L}} \sqrt[4]{\frac{\xi}{2 R}}
$$

The values of these parameters (along with the values of some others having appeared in the text) for several recent spin-wave experiments are arranged into Table II.

The ratio $M / M_{0}$ of the absolute value $M$ of the static magnetization to the equilibrium magnetization $M_{0}$ accounts for the possible higher then equilibrium polarization of the liquid. Polarizing a liquid $M / M_{0}$ times its equilibrium value increases proportionally the strength of the demagnetizing field as well as the parameters (87), (88). For the experiments [10], [18], where $M / M_{0}$ could be changed, we present in Table I the data for several integer values of $M / M_{0}$ which are close to the real experimental values.

For conditions when dipolar parameter (87) or (88) is smaller than unity one can use perturbation theory to get corrections to the modes frequencies.

As the comparison between perturbation theory based analytical calculations and numerical simulations for a sphere shows (see Fig. 3) perturbation theory has acceptable accuracy up to the values of $\omega_{M} / 2 R \nabla \omega_{L} \sim 0.1$ or $87,88 \sim 0.4$. However, at greater values of the parameters (87), (88) the discrepancy between perturbation theory and numerics grows dramatically.

When the spin wave spectra are used for measuring the transverse relaxation time $\tau$, a proper treatment of experimental data taking into account dipolar field corrections is necessary. We estimated that the dipolar restrictions on the correct determination of $\tau$ from the conventional interpretation of the spectra are of the order of the parameter (87) or (88). In particular, for the experiment $\| 10$ about $4.2 \%$. The latter means that the effect of a dipolar field can not significantly change the main conclusion of this article that the polarization induced zero temperature spin wave damping does not exist, which is in disagreement with previous spin echo experiments [6], [7], [8], [9].

A major inference for planning future experiments is the proposal to use ellipsoidal, in particular, spherical containers, not only because the estimation of the shapedependent dipolar field effects is simpler, but also because there are two roughly equal contributions to the spin wave spectrum distortion, — one from the inhomogeneity of the static demagnetizing field and the other from the demagnetizing field produced by the rotating part of the magnetization. The first contribution exists only in nonellipsoidal samples, in which a homogeneous static magnetization produces inhomogeneous demagnetizing field. For this reason implementation of such shapes for the experiments on the elicitation of the liquids characteristics from the spectra is disadvantageous.

The dependencies of (87), (88) on experimentally controllable parameters are as follows $87 \propto \omega_{L} \log \omega_{L}, \propto$ $\log \left(D_{0} / \kappa \tau_{1}\right), \propto \nabla \omega_{L}^{-1} \log \nabla \omega_{L}, \propto R^{-1}$, and 88$) \propto \omega_{L}^{5 / 4}$, $\propto\left(D_{0} / \kappa \tau_{1}\right)^{-1 / 4}, \propto \nabla \omega_{L}^{-3 / 4}, \propto R^{-1 / 4}$. So a bigger $R$ and a bigger gradient diminish the contribution of the demagnetizing field.

But for typical experimental conditions as one can see from Table II, strongly-polarized ${ }^{3} \mathrm{He}-{ }^{4} \mathrm{He}$ solutions never are too far beyond the regime of Silin spin waves perturbed by demagnetizing field, whereas pure ${ }^{3} \mathrm{He}$ at strong polarizations is in the "deep intermediate" regime, for which the results of Sec. V apply. In view of this, even at most favorable cell size and field gradient, pure highly-polarized ${ }^{3} \mathrm{He}$ seems to be unsuitable for a study of Silin waves spectra.

\section{Acknowledgments}

The authors are grateful to Prof. I. A. Fomin for sharing with them his preliminary view of the problem. One of us (P. K.) also thanks the staff of the CRTBT for their hospitality.

The work was financially supported by the Program "Jumelage entre ENS et Institut Landau" and the Landau Scholarship from Forschungszentrum Jülich, Germany (P. K.). 


\section{APPENDIX A: SPIN WAVES IN AN INFINITE MEDIUM IN A UNIFORM MAGNETIC FIELD}

The non-local dipolar term $\underline{\hat{n}}[\mathbf{m}]$ is known to become local in the important case of a medium infinite in two directions, with the proviso that $\mathbf{m}$ depends on only the remaining third coordinate. The direction in which $\mathbf{m}$ varies we denote as $\hat{s}$, and then the medium should be infinite in the two directions perpendicular to $\hat{s}$.

In such conditions

$$
\partial_{i} \partial_{j} \int_{V} \frac{f\left(\mathbf{r}^{\prime}\right)}{\left|\mathbf{r}-\mathbf{r}^{\prime}\right|} d^{3} \mathbf{r}^{\prime}=\hat{s}_{i} \hat{s}_{j} \partial_{s} \int \partial_{s} \hat{\mathcal{G}}_{1 \mathrm{~d}}\left(s-s^{\prime}\right) f\left(s^{\prime}\right) d s^{\prime},
$$

where by definition

$\partial_{a} \hat{\mathcal{G}}_{1 \mathrm{~d}}(a)=\int_{-\infty}^{\infty} \partial_{a} \frac{d^{2} \mathbf{r}_{\perp}^{\prime}}{\sqrt{\mathbf{r}_{\perp}^{\prime 2}+a^{2}}}=-\pi a \int_{a^{2}}^{\infty} \frac{d y}{y^{3 / 2}}=-2 \pi \operatorname{sgn} a$

Here $\mathbf{r}_{\perp}$ denotes the coordinate vector in the plane perpendicular to $\hat{s}$.

So $\underline{\underline{n}}_{i j}[f]=\hat{s}_{i} \hat{s}_{j} f$ and

$$
\underline{\widehat{n}}[\mathbf{m}]=\hat{s}(\hat{s} \mathbf{m}) .
$$

The case of an infinite medium in a uniform magnetic field (i.e. $\nabla \omega_{L}=0$ ) is the simplest. Looking for a solution of (34) in the form of a running wave

$$
\mathbf{m}(\mathbf{r})=\mathbf{m}_{0} e^{i \mathbf{k r}}
$$

we have $\hat{s}=\hat{k}$. Hence, Eq. (34) from which the components of the constant $\mathbf{m}_{0}$ are to be found, becomes a linear algebraic equation

$$
\left(\begin{array}{cc}
\widetilde{\omega}_{L}+\omega_{M} \hat{k}_{x}^{2} & -i \widetilde{\omega}+\omega_{M} \hat{k}_{x} \hat{k}_{y} \\
i \widetilde{\omega}+\omega_{M} \hat{k}_{x} \hat{k}_{y} & \widetilde{\omega}_{L}+\omega_{M} \hat{k}_{y}^{2}
\end{array}\right) \mathbf{m}_{0}=\frac{\omega_{M}}{4 \pi} \mathbf{h}_{\perp}^{e},
$$

where $\widetilde{\omega}_{L}=\omega_{L}-D k^{2}$ and $\widetilde{\omega}=\omega+i(D / C) k^{2}$.

$\mathbf{m}_{0}$ as a function of frequency has resonance at the $\omega$ which render the determinant of the matrix zero. This gives a Holstein-Primakoff type spectrum with an additional attenuation term due to dissipation $C^{-1} \neq 0$

$$
\omega=\sqrt{\left(\omega_{L}-D k^{2}\right)\left(\omega_{L}-D k^{2}+\omega_{M} \sin ^{2} \theta\right)}-i \frac{D}{C} k^{2},
$$

where $\theta$ is the angle between $\widehat{z}$ and $\mathbf{k}$.

\section{APPENDIX B: SPIN WAVE SPECTRUM}

A specimen placed into the field of an NMR coil changes its impedance in two ways. First, the inductance $L$ alters because so does the average energy of the field 23.

$$
\frac{L I^{2}}{2 c^{2}}=\frac{1}{8 \pi} \int \overline{\mathbf{H}(t, \mathbf{r}) \mathbf{B}(t, \mathbf{r})} d^{3} \mathbf{r}
$$

due to dispersion. $I$ is the current through the coil. A line over an expression here designates time average over oscillation period.

Secondly, the resistance $R$ appears owing to the dissipation of the energy of the field in the specimen

$$
R I^{2}=\frac{1}{4 \pi} \int \overline{\mathbf{H}(t, \mathbf{r}) \partial_{t} \mathbf{B}(t, \mathbf{r})} d^{3} \mathbf{r}
$$

Making use of the general solution (9)-(11) and of the expansion (24) we write

$$
\begin{aligned}
& \mathbf{H}(t)=H^{e} \widehat{z}+\mathbf{h}^{e}(t)+\mathbf{H}_{\mathrm{dip}}, \\
& \mathbf{B}(t)=\left(H^{e}+4 \pi M\right) \widehat{z}+\mathbf{h}^{e}(t)+4 \pi \mathbf{m}(t)+\mathbf{H}_{\mathrm{dip}}(. \mathrm{B} 4)
\end{aligned}
$$

In taking space integrals of mutual scalar products of different terms of $\mathbf{H}(t)$ and $\mathbf{B}(t)$ we note that those containing $\mathbf{H}_{\text {dip }}$ transform into integrals over a remote surface. Since beyond the specimen magnetization is zero, such integrals vanish.

In bilinear expressions we should write the monochromatic rf field $\mathbf{h}^{e}(t)=\mathbf{h}_{\mathrm{m}}^{e} \cos \omega t$ as $\mathbf{h}^{e}(t)=\frac{1}{2}\left(\mathbf{h}^{e}+\mathbf{h}^{e *}\right)$, where $\mathbf{h}^{e}=\mathbf{h}_{\mathrm{m}}^{e} e^{-i \omega t}$. Similarly, the rotating part of the magnetization should be written in the form $\mathbf{m}(t)=$ $\frac{1}{2}\left(\mathbf{m}+\mathbf{m}^{*}\right)$, where $\mathbf{m}=m_{\mathrm{m}} \mathrm{e}^{-i(\omega t+\varphi)}(1, i)$.

Then the impedance $Z=R-i \omega L / c^{2}$ of an NMR coil can be written in the form

$$
\begin{aligned}
Z & =Z_{0}+I^{-2} \int \overline{\mathbf{h}^{e}(t, \mathbf{r})\left(\partial_{t}-i \omega\right) \mathbf{m}(t, \mathbf{r})} d^{3} \mathbf{r} \\
& =Z_{0}-i \omega I^{-2} \int \mathbf{h}^{e *} \mathbf{m} d^{3} \mathbf{r}
\end{aligned}
$$

where $Z_{0}$ is the impedance without sample. So the change in the impedance of the coil due to sample is proportional to

$$
2 \int \mathbf{h}^{e *} \mathbf{m} d^{3} \mathbf{r} \approx\left\langle h^{e+} \mid m^{+}\right\rangle .
$$

The real part of this quantity gives the dispersion spectrum, while imaginary - absorption.

Introducing Green operator $\widehat{\mathcal{G}}_{\omega}(42)$ and normalizing, we arrive at expression (41).

\section{APPENDIX C: DIPOLAR CORRECTIONS TO MODES IN A FINITE CYLINDER}

In this Appendix we derive expression (56) for mode shifts due to dipolar field in a finite cylindrical cell.

The normalization coefficients of transversely homogeneous $n_{\rho}=m=0$ modes (55) are

$$
c_{n_{z}}^{-2}=\pi R^{2} \xi \int_{0}^{L / \xi \rightarrow \infty} \operatorname{Ai}^{2}\left(x+\alpha_{n_{z}}^{\prime}\right) d x .
$$

The upper limit may be put equal to infinity and then the last dimensionless integral is a number depending only on $n_{z}$. 
In calculating the integrals in (54) we use the expansion of the Green function in cylindrical coordinates [22, p. $140]$

$\frac{1}{\left|\mathbf{r}-\mathbf{r}^{\prime}\right|}=\sum_{m=-\infty}^{+\infty} e^{i m\left(\varphi-\varphi^{\prime}\right)} \int_{0}^{\infty} e^{-k\left|z-z^{\prime}\right|} J_{m}(k \rho) J_{m}\left(k \rho^{\prime}\right) d k$.

Integrals over $\varphi$ and $\varphi^{\prime}$ give $(2 \pi)^{2} \delta_{m 0}$. Then the integrals over $\rho$ and $\rho^{\prime}$ with $J_{0}$ give $R^{2} J_{1}^{2}(k R) / k^{2}$ so that

$$
\begin{aligned}
& \int_{V} \Psi(z) \underline{\widehat{n}}_{z z}[\Phi(z)] d^{3} \mathbf{r}= \\
& -\pi R^{2} \int_{0}^{\infty} \frac{J_{1}^{2}(k R)}{k^{2}} \int_{0}^{L} \Psi(z) \int_{0}^{L} \Phi\left(z^{\prime}\right) \partial_{z}^{2} e^{-k\left|z-z^{\prime}\right|} d z^{\prime} d z d k
\end{aligned}
$$

In the first integral in (54) $\Psi(z)=\psi_{n_{z}}^{2}(z), \Phi(z)=1$, while in the second $-\Psi(z)=\Phi(z)=\psi_{n_{z}}(z)$.

As a result of differentiating expansion (C2) we have

$$
\partial_{z}^{2} e^{-k\left|z-z^{\prime}\right|}=-2 k \delta\left(z-z^{\prime}\right)+k^{2} e^{-k\left|z-z^{\prime}\right|} .
$$

The integral of the $\delta$-functional part is the simpler, using $\int x^{-1} J_{1}^{2}(x) d x=\frac{1}{2}$, we obtain $\pi R^{2} \int_{0}^{L} \Psi(z) \Phi(z) d z$. For both integrals in (54) this gives unity.

So the $\delta$-functional part in the dipolar operator for transversely homogeneous spatial distributions gives the local slab value (52).

The second part in ( $\mathrm{C4})$ is shown below to be non-zero only for finite samples. Indeed, it yields

$$
-\pi R \int_{0}^{L} \Psi(z) \int_{0}^{L} \Phi\left(z^{\prime}\right) F\left(\frac{\left|z-z^{\prime}\right|}{R}\right) d z^{\prime} d z,
$$

where

$$
F(p)=\int_{0}^{\infty} J_{1}^{2}(x) e^{-p x} d x
$$

is the Laplace transform of $J_{1}^{2}$. Although its value can be found in tables (see, e.g. [25, formula 6.612]) for our purposes it is sufficient to know its value for small $p$, where it diverges logarithmically

$$
F\left(\frac{\left|z-z^{\prime}\right|}{R}\right) \approx-\frac{1}{\pi} \log \frac{e^{2}\left|z-z^{\prime}\right|}{8 R} .
$$

Writing for small $z$

$$
\int_{0}^{L} \log \frac{e^{2}\left|z-z^{\prime}\right|}{8 R} d z^{\prime}=L \log \frac{e L}{8 R}+z \log \frac{z}{e L}+O\left(z^{2}\right)
$$

and substituting $\frac{L-z}{\xi} \rightarrow x$ we obtain for the first integral in (54)

$$
1+\frac{L}{\pi R} \log \frac{e L}{8 R}+\frac{\Psi_{n_{z}}}{\pi} \frac{\xi}{R} \log \frac{\Theta_{n_{z}} \xi}{L e} .
$$

TABLE II: Numerical constants in the expression (56) for mode shifts due to dipolar field as functions of the mode number.

\begin{tabular}{lllllll}
\hline$n_{z}$ & 0 & 1 & 2 & 3 & 4 & 5 \\
\hline$\Phi_{n_{z}}$ & 1.1197 & 0.9377 & 0.6949 & 0.6449 & 0.5717 & 0.5443 \\
$\Xi_{n_{z}}$ & 0.5431 & 0.3525 & 0.2441 & 0.2384 & 0.2019 & 0.1989 \\
$\Psi_{n_{z}}$ & 0.6792 & 2.165 & 3.2134 & 4.109 & 4.915 & 5.659 \\
$\Theta_{n_{z}}$ & 0.9189 & 2.481 & 3.657 & 4.667 & 5.576 & 6.419 \\
\hline
\end{tabular}

And for the second integral in (54)

$$
1+\frac{2 \Phi_{n_{z}}}{\pi} \frac{\xi}{R} \log \frac{e^{2} \Xi_{n_{z}} \xi}{8 R} .
$$

Here the $n_{z}$-dependent constants

$$
\begin{aligned}
\Phi_{n_{z}} & =\frac{1}{2} \frac{\left(\int_{-\alpha_{n_{z}}^{\prime}}^{\infty} \operatorname{Ai}(x) d x\right)^{2}}{\int_{-\alpha_{n_{z}}^{\prime}}^{\infty} \operatorname{Ai}^{2}(x) d x}, \quad(\mathrm{C} 9) \\
\log \Xi_{n_{z}} & =\frac{\int_{-\alpha_{n_{z}}^{\prime}}^{\infty} \int_{-\alpha_{n_{z}}^{\prime}}^{\infty} \log \left|x-x^{\prime}\right| \operatorname{Ai}(x) \operatorname{Ai}\left(x^{\prime}\right) d x d x^{\prime}}{\left(\int_{-\alpha_{n_{z}}^{\prime}}^{\infty} \operatorname{Ai}(x) d x\right)^{2}} \\
\Psi_{n_{z}} & =\frac{\int_{-\alpha_{n_{z}}^{\prime}}^{\infty} x \operatorname{Ai}^{2}(x) d x}{\int_{-\alpha_{n_{z}}^{\prime}}^{\infty} \operatorname{Ai}^{2}(x) d x}, \quad(\mathrm{C} 11) \\
\log \Theta_{n_{z}} & =\frac{\int_{-\alpha_{n_{z}}^{\prime}}^{\infty} x \log (x) \mathrm{Ai}^{2}(x) d x}{\int_{-\alpha_{n_{z}}^{\prime}}^{\infty} x \mathrm{Ai}^{2}(x) d x}
\end{aligned}
$$

are of the order unity, as is seen from Table II, where they are calculated numerically for the first six modes.

Plugging (C7) and (C8) into (54) we get for the dipolar corrections to the modes frequencies in a finite cylinder expression (56).

\section{Dipolar error to transverse relaxation time}

We are now in a position to estimate the error in the determination of the transverse relaxation time because of the dipolar field.

As an example, we consider the experiment [10], wherein $\tau$ was obtained from the regime parameter $C$, which, in its turn, was determined from the slope of the linear fit to the dependence of modes half-widths $\Im \omega_{\alpha}$ on their position $\Re\left(\omega_{\alpha}-\omega_{L}-L \nabla \omega_{L}\right)$. In the collisionless regime in a finite cylinder, as is seen from (50) and (51), the two quantities are related through

$$
\frac{\Im \omega_{\alpha}}{\Re\left(\omega_{\alpha}-\omega_{L}-L \nabla \omega_{L}\right)} \approx \frac{1}{3 C} .
$$

We now find the dipolar correction to this value.

For finite $C^{-1}$ the scale $\xi$ should be replaced with the complex $\xi(1+i / C)^{1 / 3}$. This means that the dipolar field changes also imaginary parts of the eigenfrequencies and hence half-widths of the modes. In the collisionless 
regime $(C \gg 1)$ the imaginary part of the correction 56 equals

$\delta_{\text {dip }} \Im \omega_{n_{z}}^{\text {cylinder }}=\frac{\omega_{M}}{3 C} \frac{\xi}{\pi R}\left(\Phi_{n_{z}} \log \frac{8 R}{e^{3} \Xi_{n_{z}} \xi}+\Psi_{n_{z}} \log \frac{L}{\Theta_{n_{z}} \xi}\right)$

So the dipolar field changes the widths of the modes proportionally to $\omega_{M}$. Since for all parameters being positive the initial imaginary parts $\frac{1}{3 C} \alpha_{n_{z}}^{\prime} \xi \nabla \omega_{L}$ of the modes (50) are negative, the modes narrow down in the first approximation.

As a matter of fact, experimentally measured are not the absolute positions of the modes frequencies but rather their positions relative to each other. So modes positions in Ref. [10] were determined relatively to the position $\omega_{0}$ of the zeroth mode: not $\omega_{\alpha}$ but $\omega_{\alpha}-\omega_{0}$.

To diminish the error due to the data scattering in the value of the slope derived from the fitting, it is desirable to fix the Larmor frequency at the wall $\omega_{L}+L \nabla \omega_{L}$ which is experimentally badly determinable. So the position $\omega_{0}$ was put equal to its value $\omega_{0}^{(0)}=\omega_{L}+L \nabla \omega_{L}+\alpha_{0}^{\prime} \xi \nabla \omega_{L}$ in the absence of the dipolar field. This means that instead of $\omega_{\alpha}-\omega_{L}-L \nabla \omega_{L}$ in the denominator of C13 actually used in Ref. [10] was $\Re\left(\left(\omega_{\alpha}-\omega_{0}\right)+\alpha_{0}^{\prime} \xi \nabla \omega_{L}\right) \equiv \Re\left(\left(\omega_{\alpha}-\right.\right.$ $\left.\left.\omega_{0}\right)-\omega_{L}-L \nabla \omega_{L}+\omega_{0}^{(0)}\right)$.

Calculating the ratio of $\Im \omega_{\alpha}$ to this quantity taking into account the dipolar corrections (56), (C14) we get

$$
\begin{aligned}
\frac{1}{3 C}(1 & -\frac{\omega_{M}}{\alpha_{n_{z}}^{\prime} \xi \nabla \omega_{L}} \frac{\xi}{\pi R}\left(\Phi_{0} \log \frac{8 R}{e^{2+\Phi_{n_{z}} / \Phi_{0} \Xi_{0} \xi}}\right. \\
& \left.\left.+\Psi_{0} \log \frac{e^{1-\Psi_{n_{z}} / \Psi_{0} L}}{\Theta_{0} \xi}\right)\right) .
\end{aligned}
$$

So the error introduced to the determination of $C$ is of the order of 87 .

\section{APPENDIX D: SOLUTIONS FOR A SPHERICAL CELL IN ADIABATIC APPROXIMATION}

In this Appendix we obtain spin wave solutions in a sphere neglecting the dipolar field.

For a solution of the three-dimensional eigenvalue problem in a sphere of a radius $R$

$$
\begin{aligned}
{\left[D \partial^{2}+z \nabla \omega_{L}\right] u\left(z, \mathbf{r}_{\perp}\right) } & =\delta \omega u\left(z, \mathbf{r}_{\perp}\right), \\
\left.\partial_{r} u\right|_{r=R} & =0
\end{aligned}
$$

where we denoted $\delta \omega=\omega-\omega_{L}$ for brevity, the adiabatic approximation consists in the substitution

$$
u\left(z, \mathbf{r}_{\perp}\right)=v(z ; \rho) w(\rho) e^{i m \varphi}
$$

where $v_{n_{z}}(z ; \rho)$ are the eigenfunctions of the equation

$$
\left[D \partial_{z}^{2}+z \nabla \omega_{L}\right] v_{n_{z}}(z ; \rho)=\omega_{n_{z}}(\rho) v_{n_{z}}(z ; \rho) .
$$

The coordinate notations are represented in Fig. 7. The appropriate boundary conditions for (D4) will be discussed below.

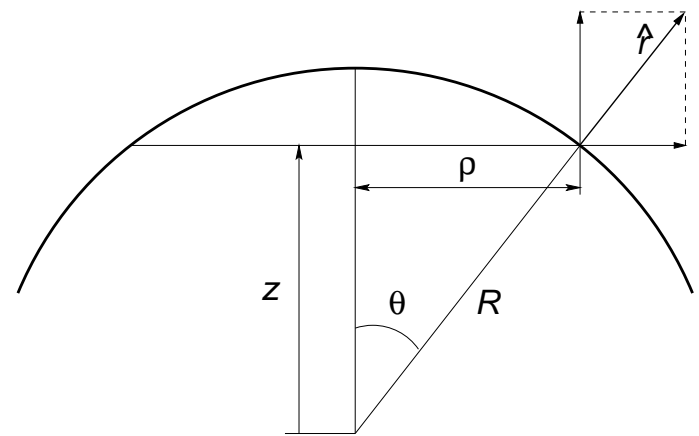

FIG. 7: A scheme of the coordinate system used to solve the eigenvalue problem in the adiabatic approximation.

Then Eq. (D1) becomes

$$
\begin{aligned}
v_{n_{z}}(z ; \rho)\left[D \partial_{\perp}^{2}+\omega_{n_{z}}(\rho)-\delta \omega\right] w(\rho) e^{i m \varphi} & \\
+D e^{i m \varphi}\left(w \partial_{\perp}^{2} v_{n_{z}}+2 \partial_{\rho} w \partial_{\rho} v_{n_{z}}\right) & =0
\end{aligned}
$$

Adiabatic approximation utilizes the fact that the second term could be for certain conditions neglected with respect to $D \partial_{\perp}^{2} w(\rho)$. Then we would obtain an effectively decoupled eigenvalue problem describing the slow transverse motion

$$
\left[D \partial_{\perp}^{2}+\omega_{n_{z}}(\rho)\right] w(\rho) e^{i m \varphi}=\delta \omega w(\rho) e^{i m \varphi} .
$$

For an estimate of the conditions of the possibility of that neglect we write

$$
\partial_{\rho} v_{n_{z}}(z ; \rho) \sim \partial_{\rho} z \partial_{z} v_{n_{z}} \sim(\rho / R) \partial_{z} v_{n_{z}},
$$

and from (D4) we see that $\partial_{z} v_{n_{z}} \sim v_{n_{z}} / \xi$, where $\xi$ is the Airy spin wave characteristic wavelength (49). On the other hand $\partial_{\rho} w \sim w / \rho$. So, the condition of applicability of the adiabatic approximation to this problem is that the ratio of the second term in (D5) to $D \partial_{\perp}^{2} w(\rho)$ should be smaller than unity

$$
\rho^{2} / R \xi \ll 1 \text {. }
$$

The boundary condition (D2) on $u$ reads

$$
\left[\cos \theta \partial_{z}+\sin \theta \partial_{\rho}\right] v_{n_{z}}(z ; \rho) w(\rho) e^{i m \varphi}=0 \text {. }
$$

For small $\theta$ we have $\cos \theta \approx 1-\rho^{2} / 2 R^{2}$ and $\sin \theta \approx \rho / R$. Estimating $\partial_{\rho} v_{n_{z}}$ as above, we see that $\sin \theta \partial_{\rho} v_{n_{z}}$ is of the order of $(\rho / R)^{2} \partial_{z} v_{n_{z}}$, and in first order in $\rho / R$ the boundary conditions are

$$
\left(w \partial_{z} v_{n_{z}}+\frac{\rho}{R} v_{n_{z}} \partial_{\rho} w\right)_{z \approx R-\frac{\rho^{2}}{2 R}}=0
$$

The first term is proportional to $1 / \xi$, while the second to $1 / R$ and in the case $\xi \ll R$ the boundary conditions reduce to a much simpler form

$$
\left.\partial_{z} v_{n_{z}}\right|_{z \approx R-\frac{\rho^{2}}{2 R}}=0
$$


Finally we formulate once again all our assumptions, i.e. $\rho, \xi, \rho^{2} / \xi \ll R$. These three reduce to $\xi \ll R$ if $\rho \sim 1 / \sqrt{a}$, where $1 / \sqrt{a}$ is the characteristic transverse spatial scale of the wave function (see Eq. (D16) below).

Now we proceed to the solution itself. First, Eq. (D4) should be solved with the boundary conditions (i) (D11) at $z=R-\rho^{2} / 2 R$ and (ii) $v_{i} \rightarrow 0$ as $z \rightarrow-\infty$.

The solution is then

$$
v_{n_{z}}(z ; \rho)=\operatorname{Ai}\left(\frac{\omega_{n_{z}}(\rho)-z \nabla \omega_{L}}{\xi \nabla \omega_{L}}\right),
$$

where

$$
\omega_{n_{z}}(\rho)=\left(R-\frac{\rho^{2}}{2 R}+\xi \alpha_{n_{z}}^{\prime}\right) \nabla \omega_{L}
$$

and $\alpha_{n}^{\prime}<0$ is the $n$-th zero of the derivative of the Airy function $\mathrm{Ai}^{\prime}$.

Then Eq. (D6) describes a two-dimensional harmonic oscillator

$$
\begin{gathered}
{\left[D \partial_{\perp}^{2}-\frac{\nabla \omega_{L}}{R} \frac{\rho^{2}}{2}\right] w(\rho) e^{i m \varphi}=} \\
\left(\omega-\left(\alpha_{n_{z}}^{\prime} \xi+R\right) \nabla \omega_{L}\right) w(\rho) e^{i m \varphi} .
\end{gathered}
$$

Solution in polar coordinates is

$$
w_{n_{\rho} m}(\rho)=\rho^{|m|} e^{-a \rho^{2} / 2} L_{n_{\rho}}^{|m|}\left(a \rho^{2}\right),
$$

where $L_{n}^{\alpha}(z)$ are the Laguerre polynomials and we denoted

$$
a^{2}=\frac{\nabla \omega_{L}}{2 D R} \equiv \frac{1}{2 \xi^{3} R}
$$

The corresponding eigenfrequencies are

$$
\omega_{n_{z} n}=\omega_{L}+R \nabla \omega_{L}+\xi \nabla \omega_{L}\left[\alpha_{n_{z}}^{\prime}-\sqrt{2 \frac{\xi}{R}}(n+1)\right],
$$

where $\omega_{L}$ is the Larmor frequency in the center of the sphere, $n=2 n_{\rho}+|m|$.

Again we remark that only the modes with zero azimuthal quantum number $m$ couple to the rf field. Eigenfunctions with $m=0$ occur for even $n=2 n_{\rho}$, as is written in (58).

\section{Effects of dipolar field in the first order of perturbation theory}

We now calculate the demagnetizing field corrections (54) to the modes in a sphere.

As we saw in the previous subsection, the dipolar-free solution of the equations of motion satisfying boundary conditions 44 is

$$
\psi_{n_{z} n_{\rho} 0}=c_{n_{z} n_{\rho} 0} \operatorname{Ai}\left(\frac{R-\frac{\rho^{2}}{2 R}-z}{\xi}+\alpha_{n_{z}}^{\prime}\right) e^{-a \rho^{2} / 2} L_{n_{\rho}}\left(a \rho^{2}\right),
$$

where $n_{z}, n_{\rho}=0,1,2, \ldots, \infty$, and $m$ was put equal to zero because only the modes with $m=0$ couple to the homogeneous rf field. Furthermore, for simplicity we consider modes with $n_{\rho}=0$ for which $L_{n_{\rho}}=1$.

In order to calculate the normalization coefficient $c_{n_{z} 00}$

$$
c_{n_{z} 00}^{-2}=2 \pi \int_{0}^{\infty} e^{-a \rho^{2}} \int_{-R+\frac{\rho^{2}}{2 R}}^{R-\frac{\rho^{2}}{2 R}} \operatorname{Ai}^{2}\left(\frac{R-\frac{\rho^{2}}{2 R}-z}{\xi}\right) d z \rho d \rho
$$

we make the substitution $x=\left(R-\rho^{2} / 2 R-z\right) / \xi$

$$
\begin{aligned}
c_{n_{z} 00}^{-2} & =2 \pi \xi \int_{0}^{\infty} e^{-a \rho^{2}} \int_{0}^{2 \frac{R-\frac{\rho^{2}}{\xi R}}{\xi}} \operatorname{Ai}^{2}\left(x+\alpha_{n_{z}}^{\prime}\right) d x \rho d \rho \\
& =\frac{\pi \xi}{a} \int_{0}^{\infty} \operatorname{Ai}^{2}\left(x+\alpha_{n_{z}}^{\prime}\right) d x
\end{aligned}
$$

Here the upper limit of integration over $x$ may be estimated as $\sim(R / \xi-1 / a R \xi)=(R / \xi-\sqrt{2 \xi / R}) \gg 1$, after which the integral over $x$ decouples from that over $\rho$ in the approximation $\xi / R \ll 1$.

In the case of a sphere $\underline{\widehat{\underline{n}}}_{z z}[1]=n^{(z)}=\frac{1}{3}$ and as was already mentioned there is no contribution to the spectrum distortion from the static dipolar field. One needs only to calculate the second integral in (54).

Again, the $\delta$-functional term in (C4) using [22]

$$
\int_{0}^{\infty} k J_{\nu}(k \rho) J_{\nu}\left(k \rho^{\prime}\right) d k=\frac{1}{\rho} \delta\left(\rho-\rho^{\prime}\right)
$$

can be easily seen to give a constant 1 as it should. In the limit $\xi / R \rightarrow 0$ a sphere transforms into a half-space, the modes being localized near the boundary. A half-space is a particular case of a slab, with the height $L \rightarrow \infty$. Hence the solutions in a sphere in the limit $\xi / R \rightarrow 0$ transform into solutions for a thick slab depending on only $z$. The demagnetizing tensor for such solutions reduces to a constant (52).

The second term in (C4) gives

$$
\begin{gathered}
\int_{V} \psi_{n_{z}}(z) \underline{\underline{n}}_{z z}\left[\psi_{n_{z}}(z)\right] d^{3} \mathbf{r}=-\pi c_{n_{z}}^{2} \xi^{2} \int_{0}^{\infty} k^{2} \int_{0}^{\infty} e^{-a \rho^{2} / 2} J_{0}(k \rho) \\
\times \int_{0}^{\infty} e^{-a \rho^{\prime 2} / 2} J_{0}\left(k \rho^{\prime}\right) \int_{0}^{\infty} \operatorname{Ai}\left(x+\alpha_{n_{z}}^{\prime}\right) \int_{0}^{\infty} \operatorname{Ai}\left(x^{\prime}+\alpha_{n_{z}}^{\prime}\right) \\
\times e^{-k \xi\left|x-x^{\prime}+\frac{\rho^{2}-\rho^{\prime 2}}{2 R \xi}\right|} d x^{\prime} d x \rho^{\prime} d \rho^{\prime} \rho d \rho d k .
\end{gathered}
$$

The two terms under the module sign appeared from $\mid z-$ $z^{\prime} \mid$. We can estimate $x-x^{\prime} \sim 1$ and $\left(\rho^{2}-\rho^{\prime 2}\right) / 2 R \xi \sim$ $1 / 2 R \xi a=\sqrt{\xi / 2 R}$, therefore $\left|z-z^{\prime}\right| \approx \xi\left|x-x^{\prime}\right|$. Then the integrals over $\rho$ and $\rho^{\prime}$ can be taken [25, formula 6.631]

$$
\int_{0}^{\infty} e^{-a \rho^{2} / 2} J_{0}(k \rho) \rho d \rho=\frac{1}{a} e^{-k^{2} / 2 a} .
$$


The integral over $k$

$$
\int_{0}^{\infty} e^{-k^{2} / a} e^{-k p} k^{2} d k
$$

where $p=\xi\left|x-x^{\prime}\right|$, is a function of $p \sqrt{a} \sim \sqrt[4]{\xi / R}$. Therefore, we can substitute (D21) with the zeroth term of its expansion in series with respect to $p \sqrt{a}$, which is $\frac{1}{4} \sqrt{\pi a^{3}}$. Then

$$
\begin{gathered}
\int_{V} \psi_{n_{z}}(z) \underline{\widehat{n}}_{z z}\left[\psi_{n_{z}}(z)\right] d^{3} \mathbf{r}=1-\frac{\Phi_{n_{z}}}{2} \sqrt{\pi a} \xi \\
=1-\frac{\Phi_{n_{z}} \sqrt{\pi}}{2} \sqrt[4]{\frac{\xi}{2 R}}
\end{gathered}
$$

where $\Phi_{n_{z}}$ are the same numbers as in the case of a cylinder.

In the end we obtain (60) for the corrections to the modes.

\section{APPENDIX E: MATRIX ELEMENTS OF THE DEMAGNETIZING OPERATOR}

In this Appendix we are going into the detail of calculation of the matrix elements of the dipolar integrodifferential operator $\underline{\widehat{n}}_{z z}$. We show that only those elements $\left\langle n l m\left|\underline{\underline{n}}_{z z}\right| n^{\prime} l^{\prime} m^{\prime}\right\rangle$ are non-zero that are between the states with $m=m^{\prime}$ and $l^{\prime}=l, l \pm 2$.

Before proceeding we remark on notations. We will write the integral operator in (14) as

$$
\hat{\mathcal{G}}_{\infty} \mathbf{M}(\mathbf{r})=\int \frac{\mathbf{M}\left(\mathbf{r}^{\prime}\right)}{\left|\mathbf{r}-\mathbf{r}^{\prime}\right|} d^{3} \mathbf{r}^{\prime}
$$

justification being that $\hat{\mathcal{G}}_{\infty}$ is the Green operator for Laplace equation with the boundary condition of vanishing at infinity.

So our plan for this section is, first, to calculate $\hat{\mathcal{G}}_{\infty}|n l m\rangle$. Then, secondly, we calculate the result of acting of $\partial_{z}^{2}$ on an arbitrary function $f(\mathbf{r})$ expanded in spherical harmonics

$$
f(\mathbf{r})=\sum_{l m} f_{l m}(r) Y_{l}^{m}(\hat{\mathbf{r}})
$$

By substituting $\hat{\mathcal{G}}_{\infty}|n l m\rangle$ for $f$ we eventually find the matrix elements themselves.

\section{Calculation of $\hat{\mathcal{G}}_{\infty}|n l m\rangle$}

We find $\hat{\mathcal{G}}_{\infty}|n l m\rangle$ directly by integration, using a wellknown formula from the theory of spherical harmonics

$$
\frac{1}{\left|\mathbf{r}-\mathbf{r}^{\prime}\right|}=\sum_{l m} \frac{4 \pi}{2 l+1} Y_{l}^{m}(\hat{\mathbf{r}}) Y_{l}^{m *}\left(\hat{\mathbf{r}}^{\prime}\right) \frac{r_{<}^{l}}{r_{>}^{l+1}},
$$

where $r_{<}\left(r_{>}\right)$is the smaller (larger) of $r$ and $r^{\prime}$. Then

$$
\begin{aligned}
\hat{\mathcal{G}}_{\infty}|n l m\rangle & =\frac{4 \pi}{2 l+1} c_{n l} Y_{l}^{m}(\hat{\mathbf{r}}) \\
& \times\left(\int_{0}^{r} \frac{r^{\prime l}}{r^{l+1}}+\int_{r}^{R} \frac{r^{l}}{r^{\prime l+1}}\right) j_{l}\left(k_{n l} r^{\prime}\right) r^{\prime 2} d r^{\prime} .
\end{aligned}
$$

The integrals at the right-hand side can be taken easily as follows. We notice that [26]

$$
\begin{aligned}
x^{-l} j_{l+1}(x) & =-\partial_{x}\left[x^{-l} j_{l}(x)\right], \\
x^{l+1} j_{l-1}(x) & =\partial_{x}\left[x^{l+1} j_{l}(x)\right] .
\end{aligned}
$$

Taking integrals from the two sides we obtain

$$
\begin{aligned}
\int_{0}^{x}\left(\frac{y}{x}\right)^{l+1} j_{l-1}(y) d y & =j_{l}(x) \\
\int_{x}^{a}\left(\frac{x}{y}\right)^{l} j_{l+1}(y) d y & =j_{l}(x)-\left(\frac{x}{a}\right)^{l} j_{l}(a) .
\end{aligned}
$$

On plugging the above into (E3) and using another property of the spherical Bessel functions

$$
j_{l+1}(x)+j_{l-1}(x)=\frac{2 l+1}{x} j_{l}(x)
$$

we get a sum of two terms

$$
\begin{aligned}
\hat{\mathcal{G}}_{\infty}|n l m\rangle & =\frac{4 \pi R^{2}}{\left(k_{n l} R\right)^{2}}|n l m\rangle \\
& -\frac{4 \pi R^{2}}{2 l+1} \frac{c_{n l}}{k_{n l} R} Y_{l}^{m}(\hat{\mathbf{r}})\left(\frac{r}{R}\right)^{l} j_{l-1}\left(k_{n l} R\right)
\end{aligned}
$$

This expression is inapplicable when $k_{n l} R=0$, which takes place for $n=l=0$. In this particular case, integrating explicitly, we get

$$
\hat{\mathcal{G}}_{\infty}|000\rangle=4 \pi c_{00} Y_{0}^{0}(\hat{\mathbf{r}}) j_{0}(0)\left(\frac{R^{2}}{2}-\frac{r^{2}}{6}\right) .
$$

\section{Calculation of $\partial_{z}^{2} f(\mathbf{r})$}

Making use of the overt expressions of the basis vectors in the spherical coordinates

$$
\begin{aligned}
\widehat{\mathbf{r}} & =\widehat{\mathbf{x}} \sin \theta \cos \varphi+\widehat{\mathbf{y}} \sin \theta \sin \varphi+\widehat{\mathbf{z}} \cos \theta \\
\widehat{\boldsymbol{\theta}} & =\widehat{\mathbf{x}} \cos \theta \cos \varphi+\widehat{\mathbf{y}} \cos \theta \sin \varphi-\widehat{\mathbf{z}} \sin \theta \\
\widehat{\boldsymbol{\varphi}} & =-\widehat{\mathbf{x}} \sin \varphi+\widehat{\mathbf{y}} \cos \varphi
\end{aligned}
$$

and of the nabla operator

$$
\boldsymbol{\partial}=\widehat{\mathbf{r}} \partial_{r}+\widehat{\boldsymbol{\theta}} \frac{1}{r} \partial_{\theta}+\widehat{\boldsymbol{\varphi}} \frac{1}{r \sin \theta} \partial_{\varphi},
$$

it can be verified that

$$
\partial_{z}=\cos \theta \partial_{r}+\frac{1}{2 r} \sin \theta\left(e^{i \varphi} \hat{l}_{-}-e^{-i \varphi} \hat{l}_{+}\right) .
$$


Here $\hat{\mathbf{l}}=-i \mathbf{r} \times \boldsymbol{\partial}$ is the angular momentum operator

$$
\hat{l}_{z}=-i \partial_{\varphi}, \quad \hat{l}_{ \pm}=e^{ \pm i \varphi}\left( \pm \partial_{\theta}+i \cot \theta \partial_{\varphi}\right),
$$

which has the well known effect on the spherical harmonics

$$
\begin{aligned}
& \hat{l}_{z} Y_{l}^{m}=m Y_{l}^{m}, \\
& \hat{l}_{+} Y_{l}^{m}=\sqrt{(l-m)(l+m+1)} Y_{l}^{m+1}, \\
& \hat{l}_{-} Y_{l}^{m}=\sqrt{(l+m)(l-m+1)} Y_{l}^{m-1} .
\end{aligned}
$$

The product of each spherical harmonic with $\sin \theta e^{i \varphi} \propto$ $Y_{1}^{1}, \cos \theta \propto Y_{1}^{0}$, or $\sin \theta e^{-i \varphi} \propto Y_{1}^{-1}$, is a sum 27]

$$
\begin{aligned}
\sin \theta e^{-i \varphi} Y_{l}^{m} & =a_{l}^{m} Y_{l+1}^{m-1}-b_{l}^{m} Y_{l-1}^{m-1}, \\
\sin \theta e^{i \varphi} Y_{l}^{m} & =-a_{l}^{-m} Y_{l+1}^{m+1}+b_{l}^{-m} Y_{l-1}^{m+1}, \\
\cos \theta Y_{l}^{m} & =c_{l+1}^{m} Y_{l+1}^{m}+c_{l}^{m} Y_{l-1}^{m}
\end{aligned}
$$

of the harmonics with the adjacent $l$ and $m$ multiplied each by a coefficient (which, in fact, are particular cases of the Clebsh-Gordan coefficients)

$$
\begin{aligned}
& a_{l}^{m}=\sqrt{\frac{l-m+1}{2 l+1} \frac{l-m+2}{2 l+3}}, \\
& b_{l}^{m}=\sqrt{\frac{l+m}{2 l+1} \frac{l+m-1}{2 l-1}}, \\
& c_{l}^{m}=\sqrt{\frac{l+m}{2 l+1} \frac{l-m}{2 l-1}} .
\end{aligned}
$$

Hence from $\mathrm{E} 12$

$$
\partial_{z} f=\sum_{l m}\left(-c_{l+1}^{m} Y_{l+1}^{m} \hat{\mathcal{L}}_{l}^{+}+c_{l}^{m} Y_{l-1}^{m} \hat{\mathcal{L}}_{l}^{-}\right) f_{l m}(r) .
$$

Here the coefficients turn out to be the same as in (E19), and we introduced two differentiating operators

$$
\begin{aligned}
& \hat{\mathcal{L}}_{l}^{+}=-\partial_{r}+l / r, \\
& \hat{\mathcal{L}}_{l}^{-}=\partial_{r}+(l+1) / r .
\end{aligned}
$$

We may rewrite (E21) by shifting the summation indices as

$$
\partial_{z} f(\mathbf{r})=\sum_{l m}\left(\partial_{z} f\right)_{l m}(r) Y_{l}^{m}(\hat{\mathbf{r}})
$$

where

$$
\left(\partial_{z} f\right)_{l m}=-c_{l}^{m} \hat{\mathcal{L}}_{l-1}^{+} f_{l-1, m}+c_{l+1}^{m} \hat{\mathcal{L}}_{l+1}^{-} f_{l+1, m} .
$$

Then repeating the procedure, we get

$$
\partial_{z}^{2} f(\mathbf{r})=\sum_{l m}\left(\partial_{z}^{2} f\right)_{l m}(r) Y_{l}^{m}(\hat{\mathbf{r}})
$$

with

$$
\begin{aligned}
\left(\partial_{z}^{2} f\right)_{l m} & =c_{l}^{m} c_{l-1}^{m} \hat{\mathcal{L}}_{l-1}^{+} \hat{\mathcal{L}}_{l-2}^{+} f_{l-2, m} \\
& +\left[\left(c_{l}^{m}\right)^{2}+\left(c_{l+1}^{m}\right)^{2}\right]\left(\partial^{2}\right)_{l} f_{l m} \\
& +c_{l+1}^{m} c_{l+2}^{m} \hat{\mathcal{L}}_{l+1}^{-} \hat{\mathcal{L}}_{l+2}^{-} f_{l+2, m} .
\end{aligned}
$$

TABLE III: Operators $\hat{\mathcal{L}}_{l}^{+}, \hat{\mathcal{L}}_{l}^{-}$.

\begin{tabular}{lllll}
\hline$f(r)$ & $r^{l}$ & $1 / r^{l+1}$ & $j_{l}(r)$ & $j_{l}($ const $r)$ \\
\hline$\hat{\mathcal{L}}_{l}^{+} f$ & 0 & $(2 l+1) f / r$ & $j_{l+1}(r)$ & const $j_{l+1}(\operatorname{const} r)$ \\
$\hat{\mathcal{L}}_{l}^{-} f$ & $(2 l+1) f / r$ & 0 & $j_{l-1}(r)$ & const $j_{l-1}($ const $r)$ \\
\hline
\end{tabular}

Here we used that $\hat{\mathcal{L}}_{l-1}^{+} \hat{\mathcal{L}}_{l}^{-}=\hat{\mathcal{L}}_{l+1}^{-} \hat{\mathcal{L}}_{l}^{+}=-\left(\partial^{2}\right)_{l}$, where $\left(\partial^{2}\right)_{l}$ is the $l$-th component of the Laplace operator

$$
\left(\partial^{2}\right)_{l}=\partial_{r}^{2}+2 \frac{\partial_{r}}{r}-\frac{l(l+1)}{r^{2}} .
$$

Changing the summation indices in each term again we arrive at

$$
\begin{aligned}
\partial_{z}^{2} f= & \sum_{l m}\left(c_{l+1}^{m} c_{l+2}^{m} Y_{l+2}^{m}(\hat{\mathbf{r}}) \hat{\mathcal{L}}_{l+1}^{+} \hat{\mathcal{L}}_{l}^{+}\right. \\
& +\quad\left[\left(c_{l}^{m}\right)^{2}+\left(c_{l+1}^{m}\right)^{2}\right] Y_{l}^{m}(\hat{\mathbf{r}})\left(\partial^{2}\right)_{l} \\
& \left.+\quad c_{l-1}^{m} c_{l}^{m} Y_{l-2}^{m}(\hat{\mathbf{r}}) \hat{\mathcal{L}}_{l-1}^{-} \hat{\mathcal{L}}_{l}^{-}\right) f_{l m}
\end{aligned}
$$

\section{Matrix elements}

Substituting $\hat{\mathcal{G}}_{\infty}|n l m\rangle$ from (E9) into (E26) and using the properties of $\hat{\mathcal{L}}_{l}^{+}, \hat{\mathcal{L}}_{l}^{-}$summarized in Table III, we find

$$
\begin{aligned}
\frac{1}{4 \pi} \partial_{z}^{2} \hat{\mathcal{G}}_{\infty}|n l m\rangle & =c_{l+1}^{m} c_{l+2}^{m} c_{n l} j_{l+2}\left(k_{n l} r\right) Y_{l+2}^{m}(\hat{\mathbf{r}}) \\
& -\left[\left(c_{l}^{m}\right)^{2}+\left(c_{l+1}^{m}\right)^{2}\right]|n l m\rangle \\
& +c_{l-1}^{m} c_{l}^{m} c_{n l} Y_{l-2}^{m}(\hat{\mathbf{r}})\left(j_{l-2}\left(k_{n l} r\right)\right. \\
& \left.-(2 l-1) \frac{j_{l-1}\left(k_{n l} R\right)}{k_{n l} R}\left(\frac{r}{R}\right)^{l-2}\right) .
\end{aligned}
$$

It is not hard to verify by straightforward differentiating that this expression holds also for $n=l=m=0$.

The matrix elements $\left\langle n^{\prime} l^{\prime} m^{\prime}\left|\partial_{z}^{2} \hat{\mathcal{G}}_{\infty}\right| n l m\right\rangle$ are to be obtained from this expression by integrating with the complex conjugate of (79) over the spherical volume of our sample. In doing so we see that only the elements with $m^{\prime}=m, l^{\prime}=l, l \pm 2$ are non-zero as we already mentioned in the text

$$
\begin{aligned}
\langle n l m| \frac{1}{4 \pi} & \partial_{z}^{2} \hat{\mathcal{G}}_{\infty}\left|n^{\prime}, l^{\prime}=l-2, m\right\rangle \\
= & \left(c_{l-1}^{m} c_{l}^{m}\right) \int_{0}^{R} c c^{\prime} j_{l}(k r) j_{l}\left(k^{\prime} r\right) r^{2} d r \\
\langle n l m| \frac{1}{4 \pi} & \partial_{z}^{2} \hat{\mathcal{G}}_{\infty}\left|n^{\prime} l m\right\rangle=-\left[\left(c_{l}^{m}\right)^{2}+\left(c_{l+1}^{m}\right)^{2}\right] \delta_{n n^{\prime}} \\
\langle n l m| \frac{1}{4 \pi} & \partial_{z}^{2} \hat{\mathcal{G}}_{\infty}\left|n^{\prime}, l^{\prime}=l+2, m\right\rangle \\
= & \left(c_{l^{\prime}-1}^{m} c_{l^{\prime}}^{m}\right) c c^{\prime}\left(\int_{0}^{R} j_{l}(k r) j_{l}\left(k^{\prime} r\right) r^{2} d r\right. \\
& \left.-(2 l+3) R^{3} \frac{j_{l+1}(k R)}{k R} \frac{j_{l+1}\left(k^{\prime} R\right)}{k^{\prime} R}\right)
\end{aligned}
$$


where we introduced shorthand notations $k=k_{n l}, k^{\prime}=$ $k_{n^{\prime} l^{\prime}}, c=c_{n l}, c^{\prime}=c_{n^{\prime} l^{\prime}}$.

In the last term we used

$$
\int_{0}^{R} j_{l}(k r) r^{l+2} d r / R^{l}=R^{3} j_{l+1}(k R) / k R
$$

with $k=k_{n l}$. There is one exception, though, when $l=n=0$ - then $k_{n l}=k_{00}=0$ and the division in the right-hand side of (E29) is undefined. Integrating straightforwardly, we get instead $R^{3} j_{0}(0) / 3$.

At the end, we take integrals in the off-diagonal elements of (E28) using the formulas below 25, formula $5.54]$

$$
\begin{aligned}
\int j_{l}(a x) j_{l}(b x) x^{2} d x \\
=x^{2} \frac{b j_{l-1}(b x) j_{l}(a x)-a j_{l-1}(a x) j_{l}(b x)}{a^{2}-b^{2}} \\
\equiv x^{2} \frac{-b j_{l+1}(b x) j_{l}(a x)+a j_{l+1}(a x) j_{l}(b x)}{a^{2}-b^{2}} \\
\equiv x^{2} \frac{b j_{l+1}(b x) j_{l+2}(a x)-a j_{l+1}(a x) j_{l+2}(b x)}{a^{2}-b^{2}} \\
\quad+(2 l+3) x^{3} \frac{j_{l+1}(a x)}{a x} \frac{j_{l+1}(b x)}{b x} .
\end{aligned}
$$

Here $a \neq b$ and we consecutively applied recurrent rela- tion (E8) first to $j_{l-1}$ and then to $j_{l}$.

Plugging the integration limits we see that abovediagonal $\left(l^{\prime}=l+2\right)$ elements equal corresponding belowdiagonal $\left(l^{\prime}=l-2\right)$ with the appropriate change $l^{\prime} \leftrightarrow l$. (For $n=l=0$ this is to be verified manually.)

Finally, we get formula (84) in the text for the dipolar part of the Hamiltonian.

The case $a=b$ in $(\mathbb{E} 30)$ is used in the calculation of the normalization coefficients $c_{n l} 80$

$$
\int j_{l}^{2}(a x) x^{2} d x=\frac{x^{3}}{2}\left(j_{l}^{2}(a x)-j_{l-1}(a x) j_{l+1}(a x)\right)
$$

We get

$$
\begin{aligned}
c_{n l} & =\left(\frac{R^{3}}{2}\left[j_{l}^{2}\left(k_{n l} R\right)-j_{l-1}\left(k_{n l} R\right) j_{l+1}\left(k_{n l} R\right)\right]\right)^{-1 / 2}, \\
& =\left(\frac { R ^ { 3 } } { 2 } \left[j_{l}^{2}\left(k_{n l} R\right)+j_{l+1}^{2}\left(k_{n l} R\right)\right.\right. \\
& \left.\left.-\frac{2 l+1}{k_{n l} R} j_{l}\left(k_{n l} R\right) j_{l+1}\left(k_{n l} R\right)\right]\right)^{-1 / 2},
\end{aligned}
$$

when $n+l \geq 1$. For $n=l=0$ we have $k_{00}=0$ and $j_{l-1}$ grows unlimitedly when its argument tends to zero. Then we integrate directly $c_{00}=\left(R^{3} j_{0}^{2} / 3\right)^{-1 / 2}$.
[1] A. E. Meyerovich, Phys. Lett. A107, 177 (1985).

[2] J. W. Jeon and W. J. Mullin, J. Phys. (Paris) 49, 1691 (1988); Phys. Rev. Lett. 62, 2691 (1989); W. J. Mullin and J. W. Jeon, J. Low Temp. Phys. 88, 433 (1992).

[3] A. E. Meyerovich and K. A. Musaelian, J. Low Temp. Phys. 89, 781 (1992); J. Low Temp. Phys. 94, 249 (1994); J. Low Temp. Phys. 95, 789 (1994); Phys. Rev. Lett. 72, 1710 (1994).

[4] D. I. Golosov and A. E. Ruckenstein, Phys. Rev. Lett., 74, 1613 (1995); J. Low Temp. Phys. 112, 265 (1998).

[5] I. A. Fomin, JETP Lett., 65, 749 (1997).

[6] D. Candela, D. R. McAllaster, L-J. Wei, and N. Kalechofsky, J. Low Temp. Phys. 89, 307 (1992); L.-J. Wei, N. Kalechofsky and D. Candela, Phys. Rev. Lett. 71, 879 (1993).

[7] D. Candela, H. Akimoto, R. M. Bowley, O. Buu, D. Clubb, J. R. Owers-Bradley, J. Low Temp. Phys. 121, 767 (2000).

[8] J. H. Ager, A. Child, R. Konig et al., J. Low Temp. Phys. 99, 683 (1995).

[9] J. R. Owers-Bradley, R. M. Bowley, O. Buu, D. Clubb and G. Vermeulen, J. Low Temp. Phys. 121, 779 (2000).

[10] G. A. Vermeulen, A. Roni, Phys. Rev. Lett. 86, 248 (2001).

[11] D. S. Golubev and A. D. Zaikin, Phys. Rev. Lett. 81, 1074 (1998), Phys. Rev. B 59, 9195 (1999).

[12] I. L. Aleiner, B. L. Altshuler, M. E. Gershenson, Phys. Rev. Lett. 82, 3190 (1999).

[13] D. Cohen, J. Imry, Phys. Rev. B 59, 11143 (1999).
[14] Ch. Kittel, Phys. Rev. 71, 270 (1947), Phys. Rev. 73, 155 (1948).

[15] L. R. Walker, Phys. Rev. 105, 390 (1957), J. Appl. Phys. 29, 318 (1958).

[16] D. Candela, N. Masuhara, D. S. Sherill, and D. O. Edwards, J. Low Temp. Phys. 63, 369 (1986).

[17] D. Candela, D. R. McAllaster, and L-J. Wei, Phys. Rev. B 44, 7510 (1991).

[18] A. Roni and G. Vermeulen, Physica B280, 87 (2000); A. Roni, PhD thesis, Université Joseph FourierGrenoble I, 1999, in French (unpublished, available in pdf format from www-crtbt.polycnrs-gre.fr/he3pold dilution/spinwaves.html).

[19] For susceptibility of the saturated dilute phase we used table II of A. Rodriguez and G. Vermeulen, J. Low Temp. Phys. 108, 103 (1997) based on the susceptibility data of A. I. Ahonen, M. A. Paalanen, R. C. Richardson, and Y. Takano, J. Low Temp. Phys. 24, 733 (1976) and molar volume and concentration data of G. E. Watson, J. D. Reppy, and R. C Richardson, Phys. Rev. 188, 384 (1968). For the susceptibility of pure ${ }^{3} \mathrm{He}$, we used a fit to H. Ramm, P. Pedroni, J. R. Thompson, and H. Meyer, J. Low Temp. Phys. 2, 539 (1970) and the molar volume given by Greywall, Phys. Rev. B33, 7520 (1986). The zero-temperature susceptibility of the very dilute mixtures is calculated from $m^{*}$ and $T_{\mathrm{F}}$ written in Ref. 17]. A susceptibility at a finite temperature then was read from the temperature dependence in Fig. 4 of the same reference. And molar volume data were taken from Watson 
et al. Spin diffusion data are taken from the respective sources, for pure ${ }^{3} \mathrm{He}$ at 8 bar in 18 we used the spin diffusion data for 6.3 bar from 16$]$.

[20] A. J. Leggett, J. Phys. C 3, 448 (1970). Our notation of $\mathbf{M}$ as magnetization - the local average macroscopic magnetic moment density - differs from Leggett's $\mathbf{M}$ as the local spin density by the factor $\gamma \hbar$. The same holds for the current.

[21] In Leggett's paper the external magnetic field $\mathbf{H}^{e}$ entered the equations (2), (3) instead of B. Strictly speaking, it should be the exact microscopic field $\mathbf{h}$, but the particles motion with ranges greater than interatomic distances leads to its averaging, giving magnetic flux B. See discussion at the end of $\S 62$ of L. D. Landau, E. M. Lifshitz and L. P. Pitaevskii, Course of Theoretical Physics, 2nd edition, Vol. IX, Pergamon Press, 1980.

[22] J. D. Jackson, Classical Electrodynamics, 3rd edition, Wiley, NY, 1999.

[23] L. D. Landau, E. M. Lifshitz and L. P. Pitaevskii, Course of Theoretical Physics, 2nd edition, Vol. VIII, Chapter
IX, Pergamon Press, 1984.

[24] P. M. Morse and H. Feshbach, Methods of Theoretical Physics, Vol. I, McGraw-Hill, NY, 1953.

[25] I. S. Gradshteyn and I. M. Ryzhik, Tables of Integrals, Series and Products, 5th edition, Academic Press, 1994.

[26] M. Abramovitz and I. A. Stegun, ed., Handbook of Mathematical Functions, 9th edition, Dover, NY, 1972.

[27] E. Merzbacher, Quantum Mechanics, 2nd edition, Wiley, NY, 1970.

[28] The original results of deWames and Wolfram were published rather fragmentarily in several papers. A good review of the work and all the references may be found in a review article by C. E. Patton, Phys. Rep. 103, 251 (1984).

[29] G. Deville, M. Bernier, and J. M. Delrieux, Phys. Rev. B 19, 5666 (1979).

[30] I. A. Fomin and G. A. Vermeulen, J. Low Temp. Phys. 106, 133 (1997). 